\title{
Bifurcations of Circle Maps: Arnol'd Tongues, Bistability and Rotation Intervals
}

\author{
Philip L. Boyland* \\ Department of Mathematics, Boston University, Boston, MA 02115, USA
}

\begin{abstract}
We study the bifurcations of two parameter families of circle maps that are similar to $f_{b, w}(x)=x+w+(b / 2 \pi) \sin (2 \pi x)(\bmod 1)$. The bifurcation diagram is constructed in terms of sets $T_{r}$, where $T_{r}$ is the set of parameter values $(b, w)$ for which $f_{b, w}$ has an orbit with rotation number $r$. We show that the known structure when $b<1$ (for $r$ rational, $T_{r}$ is an Arnol'd tongue and for $r$ irrational, it is an arc) extends nicely into the region $b>1$, where $f_{b, w}$ is no longer injective and can have an interval of rotation numbers. Specifically, the tongues overlap in a uniform, monotonic manner and for $r$ irrational, $T_{r}$ opens into a tongue. Our other theorems give information about the dynamics of $f_{b, w}$ (e.g., bistability or aperiodicity) for $(b, w)$ in various subsets of parameter space.
\end{abstract}

\section{Introduction}

In this paper we construct the bifurcation diagram of two parameter families of circle maps which are similar to what has been termed the "canonical" family [16], $f_{b, w}(x)=x+w+(b / 2 \pi) \sin (2 \pi x)(\bmod 1)$. These families are used to model various periodically forced nonlinear oscillators (e.g. $[8,17,23])$ and in the renormalization group analysis of the transition from quasiperiodicity to chaos $[9,22,24])$. When $b<1, f_{b, w}$ is a $C^{\infty}$ circle diffeomorphism. The dynamics of such maps are well understood from the classic work of Poincaré and Denjoy. All orbits rotate at the same asymptotic rate, so one may define the rotation number of the diffeomorphism which essentially classifies the dynamics. When $b>1$ however, $f_{b, w}$ is no longer injective and can display the dynamic complexity which is well known for maps of the interval. Different orbits can rotate at different asymptotic rates, and $f_{b, w}$ can have an interval of rotation numbers [18].

Of particular interest in the bifurcation theory of dynamical systems is the description of the transition from simple to complicated or "chaotic" dynamics. In studying this transition in the canonical family it is natural to examine the

* Current address: Department of Mathematics, Maharishi International University, Fairfield, IA 52556, USA 
bifurcation diagram in terms of the sets $T_{r}$, where $T_{r}$ is the set of parameter values $(b, w)$ for which $f_{b, w}$ has an orbit with rotation number $r$. The manner in which these sets overlap and accumulate indicates how the rotational dynamics are changing as $b$ and $w$ are varied.

The structure of the $T_{r}$ when $b<1$ is given by Arnol'd [1] and Herman [15] who use the canonical family as an example. When $r$ is rational, $T_{r}$ is a tongue shaped region with its narrow tip on the line $b=0$, while for $r$ irrational, $T_{r}$ is an arc connecting the line $b=0$ to the line $b=1$ (see Fig. 1). We prove that this structure extends nicely into the region where $b>1$. Specifically, the tongues overlap in a uniform, monotonic manner and for $r$ irrational, $T_{r}$ opens out into a tongue with its tip on the line $b=1$ (see Fig. 1).

Our other main theorems describe finer structure of the bifurcation diagram within $T_{p / q}$ for $p / q$ an arbitrary rational. For $(b, w)$ in these various subsets of $T_{p / q}, f_{b, w}$ has such dynamical behavior as a superstable periodic orbit, a stable periodic orbit, a saddle node or a homoclinic orbit, all with rotation number $p / q$.

As a corollary, we delineate the ways in which $f_{b, w}$ can make the transition from having a single rotation number to having an interval of rotation numbers. It is also shown that for each pair of rationals $r_{2}>r_{1}$, there is an open set $U$ so that $(b, w) \in U$ implies that $f_{b, w}$ has exactly two stable periodic orbits $z_{1}$ and $z_{2}$ with rotation numbers $r_{1}$ and $r_{2}$ respectively (i.e. "bistability"). Finally, we show that if the set of rotation numbers of $f_{b, w}$ is the interval $[\alpha, \beta]$ with $\alpha$ and $\beta$ irrational, then $f_{b, w}$ has no stable periodic orbits (i.e. is "aperiodic").

We have been especially guided in our study by the numerical work and description of the canonical family by Glass and his various coworkers (sum-

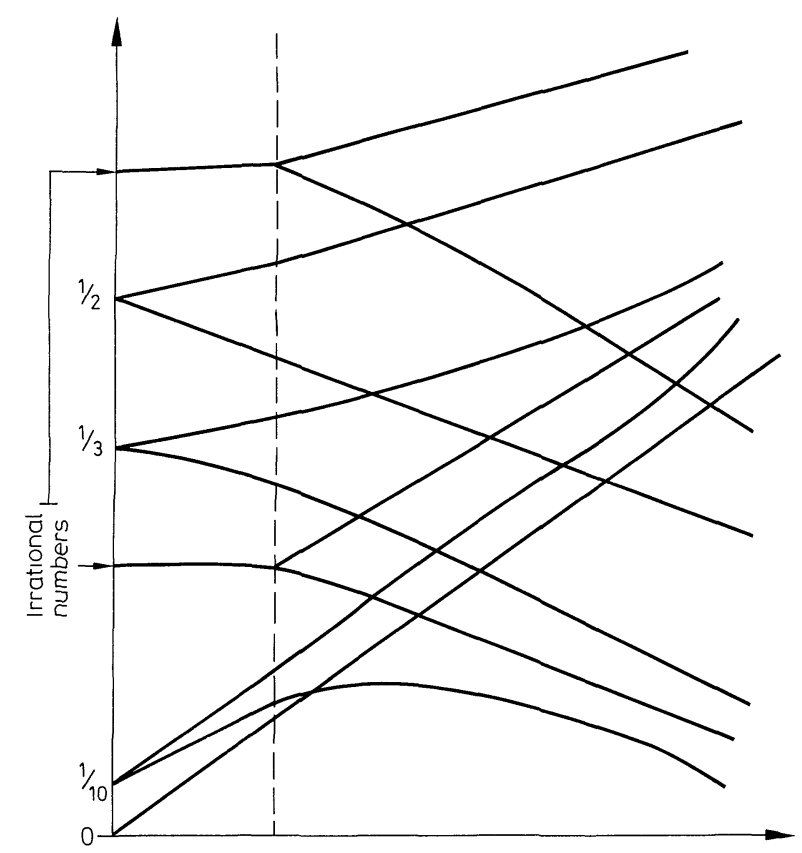

Fig. 1. Sketch of the bifurcation diagram of the canonical family 
marized in [10]). Our theorems give analytic proof to many of their descriptions and conjectures. Our other source of motivation and guidance was the bifurcation diagram proposed by Aronson, Chory, Hall, and McGehee in their computer assisted study of bifurcations from an invariant circle in families of plane diffeomorphisms [2]. We shall see many interesting similarities between the bifurcation diagram of the canonical family and the diagram proposed by Aronson et al. (A detailed description of the possible relationship of the bifurcation diagram of the canonical family to the phenomenon of the breakup of a mode locked invariant circle is contained in the appendix of [22].) We wish to emphasize, however, that the bifurcation structure of a family of circle maps is certainly inadequate to explain all the diverse phenomena that can accompany the loss of an invariant circle in a family of plane diffeomorphisms (e.g. infinitely many sinks). We also note that a bifurcation diagram similar to that proposed by Aronson et al. occurs in a somewhat different context in Greenspan and Holmes' study of a periodically forced Duffing equation [11].

This paper is organized as follows. In the first section we present some generalizations to noninjective circle maps of some well known results about circle homeomorphisms. The second section is devoted to the study of a particularly simple form of noninjective circle map. Each noninjective member of the two parameter families we will study is of this type. With Sect. 3, we commence our study of two parameter families of circle maps of the form $f_{b, w}(x)=x+w$ $+b p(x)(\bmod 1)$, where $p(x)$ satisfies hypotheses which make it qualitatively similar to $(1 / 2 \pi) \sin (2 \pi x)$. It turns out that one only needs $p(x)$ continuous to construct the overall organization of the bifurcation diagram. In Sects. 3 and 4 we obtain more information about the bifurcation diagram and the corresponding dynamics of $f_{b, w}$ by requiring $p(x)$ to be increasing like $(1 / 2 \pi) \sin (2 \pi x)$. In Sect. $4, p(x)$ is $C^{1}$, and in Sect. 5 we require $f_{b, w}$ to have negative Schwarzian derivative for all $b>1$.

\section{Rotation Intervals of Degree One Circle Maps}

In this first section we introduce notation and collect together some lemmas concerning the set of rotation numbers of degree one circle maps that will be useful in our study of two parameter families. These results are straightforward generalizations of known results concerning the rotation number of circle homeomorphisms contained in Arnol'd [1], Brunovsky [6], and Herman [16].

We begin with some standard definitions. For a function $f: S^{1} \rightarrow S^{1}$, define the $n^{\text {th }}$ iterate of $f, f^{n}=f \circ f^{n-1}$. The orbit of $x$ under $f$ is defined as $o(x, f)$ $=\left\{x, f(x), f^{2}(x), \ldots\right\}$. The rotation number of $x$ under $f, \varrho(x, f)$, measures the asymptotic rate of rotation of an orbit. It was first defined by Poincare for circle homeomorphisms and was generalized to degree one circle maps by Newhouse, Palis, and Takens as follows [21].

For $f: S^{1} \rightarrow S^{1}$ a degree one map, fix a lift $F: \mathbb{R} \rightarrow \mathbb{R}$, i.e. $\pi \circ F=F \circ \pi$, where $\pi: \mathbb{R} \rightarrow S^{1}$ is $\pi(t)=\exp (2 \pi i t)$. Choose $x^{\prime}$ with $\pi\left(x^{\prime}\right)=x$ and define

$$
\varrho(x, f)=\varrho\left(x^{\prime}, F\right)=\limsup _{n \rightarrow \infty} \frac{F^{n}\left(x^{\prime}\right)-x^{\prime}}{n} .
$$


Next, define the rotation set of $f$ as $\varrho(f)=\left\{\varrho(x, f): x \in S^{1}\right\}$. If we had chosen a different lift $F$, the entire rotation set could be translated by an integer. Poincaré showed that if $f$ is a homeomorphism, $\varrho(x, f)$ is independent of the choice of $x$, and so $\varrho(f)$ is a single number, called the rotation number of $f$. If $f$ is a continuous degree one circle map, Ito [18] (see also [21]) has shown that $\varrho(f)$ is a closed interval which we denote $\left[\varrho_{1}(f), \varrho_{2}(f)\right]$.

Any map $F: \mathbb{R} \rightarrow \mathbb{R}$ which satisfies $F(x+1)=F(x)+1$ may be treated as the lift of a degree one circle map. In what follows, it will usually be more convenient to work with lifts of circle maps rather than the circle maps themselves. We note for future use that $\varrho\left(x, F^{q}\right)=q \varrho(x, F)$. The proof is straightforward. As above, we may write, $\varrho(F)=\left[\varrho_{1}(F), \varrho_{2}(F)\right]$.

The first lemma introduces some simple relationships between the lift of a rigid rotation $R_{r}\left(R_{r}(x)=x+r\right)$, the lift of a degree one circle map $F$, and $\varrho_{1}(F)$ and $\varrho_{2}(F)$. The proof is standard. We include it because of the importance of these techniques in the sequel. As will be the case for many of our lemmas and theorems, the statement will involve both $\varrho_{1}$ and $\varrho_{2}$, but we will only prove the result for $\varrho_{1}$, the proof for $\varrho_{2}$ being completely analogous.

Lemma 1.1. $F$ is the lift of a degree one circle map, $r$ is a real number and $p$ and $q$ are integers with $(p, q)=1$.

(a) If $F \geqq R_{r}$ (respectively, $\left.F \leqq R_{r}\right)$, then $\varrho_{1}(F) \geqq r\left(\varrho_{2}(F) \leqq r\right)$.

(b) If $F>R_{r}\left(F<R_{r}\right)$, then $\varrho_{1}(F)>r\left(\varrho_{2}(F)<r\right)$.

(c) $F^{q}>R_{p}\left(F^{q}<R_{p}\right)$ if and only if $\varrho_{1}(F)>p / q\left(\varrho_{2}(F)<p / q\right)$.

Proof. We prove (b) first. Since $F(x+1)=F(x)+1, F(x)-x$ is periodic and thus has compact image. So we may find an $\varepsilon>0$ with $F(x)>x+r+\varepsilon$ for all $x$ in $R$. Using induction this implies that $F^{n}(x)>x+n(r+\varepsilon)$ or

$$
\frac{F^{n}(x)-x}{n}>r+\varepsilon
$$

for all $n$ and all $x$ in $\mathbb{R}$.

The proof of (a) is similar. To prove (c), note that $F^{q}>R_{p}$ implies by (b) that $\varrho_{1}\left(F^{q}\right)>p$. Thus $\varrho_{1}(F)>p / q$. For the converse, assume there exists a $z$ with $F^{q}(z)$ $\leqq z+p$. Then using continuity, either $F^{q}<R_{p}$ in which case $\varrho_{1}(F) \leqq \varrho_{2}(F)<p / q$, or there is a point $x$ with $F^{q}(x)=x+p$ so $\varrho(x)=p / q$, and so $p / q \in \varrho(f)$. Thus $\varrho_{1}(F)$ $\leqq p / q$.

Throughout the remainder of this work, $p$ and $q$ will always be integers with $(p, q)=1$. A point $z$ belongs to a $p / q$-periodic orbit under $f: S^{1} \rightarrow S^{1}$ if $\varrho(z, f)=p / q$ and $z$ is periodic with least period $q$.

The next two lemmas are contained in Newhouse-Palis-Takens [21]. The proofs are straightforward applications of Lemma 1.1.

Lemma 1.2. $f: S^{1} \rightarrow S^{1}$ is degree one and continuous. $F$ is its lift satisfying $F(0) \in[0,1)$. The following are equivalent:

(a) $p / q \in \varrho(f)$,

(b) $f$ has a p/q-periodic point $z$,

(c) There is a point $z$ with $F^{q}(z)=z+p$. 
It has been known since Poincare that the rotation number of a circle homeomorphism depends continuously on the function. The first published proof was apparently given by Arnol'd [1]. The next result generalizes this to the rotation interval of degree one circle maps. Note, however, that simple examples show that $\varrho(x, f)$ is not continuous in either $x$ or $f$. Let End $\left(S^{1}\right)$ denote the set of all continuous, degree one circle maps with the $C^{0}$-topology.

Lemma 1.3. $\varrho=\left(\varrho_{1}, \varrho_{2}\right): \operatorname{End}\left(S^{1}\right) \rightarrow \mathbb{R}^{2}$ is continuous.

We remark that in Lemma 1.3 we must choose lifts that are $C^{0}$-close when computing the rotation intervals of circle maps that are $C^{0}$-close. Now let

$$
E_{p / q}=\left\{f \in \operatorname{End}\left(S^{1}\right): p / q \in \varrho(f)\right\} .
$$

Note that

$$
\left.E_{p / q}=\varrho^{-1}\left(\{u, v) \in \mathbb{R}^{2}: u \leqq p / q \leqq v\right\}\right)
$$

and is thus closed. The statement " $F^{q} \geqq R_{p}$ with equality attained" means that $F^{q} \geqq R_{p}$ and there is a point $z$ with $F^{q}(z)=z+p$.

Lemma 1.4. If $f \in B d\left(E_{p / q}\right)$, then either $F^{q} \geqq R_{p}$, or $F^{q} \leqq R_{p}$ with equality attained in both cases. In the first case, $\varrho_{1}(f)=p / q$ and in the second, $\varrho_{2}(f)=p / q$.

Proof. Since $E_{p / q}$ is closed, $f \in B d\left(E_{p / q}\right)$ implies that $p / q \in \varrho(f)$. Thus $F^{q}>R_{p}$ and $F^{q}<R_{p}$ are impossible by Lemma 1.2. So if the conclusion of the lemma does not hold, there are points $z_{1}$ and $z_{2}$ with $F^{q}\left(z_{1}\right)>z_{1}+p$ and $F^{q}\left(z_{2}\right)<z_{2}+p$. But these inequalities will also hold with $G$ in place of $F$ for any $g C^{0}$-close to $f$. Thus there is a point $x$ (depending on $g$ ) with $G^{q}(x)=x+p$. Therefore, $p / q \in \varrho(g)$ for any $g$ $C^{0}$-close to $f$ so $f \notin B d\left(E_{p / q}\right)$.

To prove the second statement of the lemma, since $p / q \in \varrho(f), \varrho_{1}(f) \leqq p / q$ $\leqq \varrho_{2}(f)$. Using Lemma $1.1(\mathrm{c})$, if $F^{q} \geqq R_{p}, \varrho_{1}(f) \geqq p / q$, so $\varrho_{1}(f)=f / q$. Similarly, $F^{q} \leqq R_{p}$ implies $\varrho_{2}(f) \leqq p / q$, so $\varrho_{2}(f)=p / q$ in this case also.

One interpretation of Lemma 1.4 is that the bifurcation which occurs when $p / q$ is lost as a rotation number is always a $p / q$-saddle node (perhaps degenerate).

The next two lemmas generalize some well known results about circle homeomorphisms to nondecreasing maps, $\left[f: S^{1} \rightarrow S^{1}\right.$ is called nondecreasing if its lift $F$ has the property that $x<y$ implies $F(x) \leqq F(y)]$. Their statements are essentially contained in Hall [12]. The proofs are only slight modifications of the proofs for homeomorphisms and Hall refers the reader to Herman [16]. The first lemma states that nondecreasing, degree one circle maps have a single rotation number.

Lemma 1.5. If $h: S^{1} \rightarrow S^{1}$ is continuous, nondecreasing and degree one, then

$$
\lim _{n \rightarrow \infty} \frac{H^{n}(x)-x}{n}
$$

exists and is independent of $x$.

Lemma 1.6. F, $G$, and $H$ are lifts of continuous degree one circle maps with $G$ and $H$ nondecreasing. 
(a) If $G \leqq H$, then $G^{q} \leqq H^{q}$ for all positive integers $q$, and so $\varrho(G) \leqq \varrho(H)$.

(b) If $G<H$, then $G^{q}<H^{q}$ for all positive integers $q$, and if either $\varrho(G)$ or $\varrho(H)$ is irrational, $\varrho(G)<\varrho(H)$.

(c) If $F \geqq H$, then $F^{q} \geqq H^{q}$ for all positive integers $q$, and so $\varrho_{1}(F) \geqq \varrho(H)$. If $F \leqq H, F^{q} \leqq \bar{H}^{q}$ for all positive integers $q$, and so $\varrho_{2}(F) \leqq \varrho(H)$.

Simple examples show that conclusion (c) may be false if $H$ is not nondecreasing.

\section{A Class of Circle Endomorphisms}

In this section we study a class of noninjective circle maps denoted by $A$. Each member of the two parameter families we will eventually study will be in this class. A circle map $g$ is in $A$ if $g$ is continuous, degree one and its lift $G$ is piecewise strictly monotone with precisely two turning points in the interval $(0,1)$, a maximum $m_{2}$ and a minimum $m_{1}$ with $m_{1}>m_{2}$ (see Fig. 2). This class has been studied by Bernhardt [3] from a point of view different from the one we shall pursue.

The following construction is due to Hall [13]. ${ }^{1}$ It will be very useful in providing a geometric tool for working with the rotation interval. For a fixed $g \in A$ and $\mu \in[1,2]$, there are unique points $z_{1, \mu} \in\left[m_{1}, 1\right]$ and $z_{2, \mu} \in\left[0, m_{2}\right]$ with

$$
G\left(z_{1, \mu}\right)=G\left(z_{2, \mu}\right)=G\left(m_{1}\right)+(\mu-1)\left(G\left(m_{2}\right)-G\left(m_{1}\right)\right)
$$

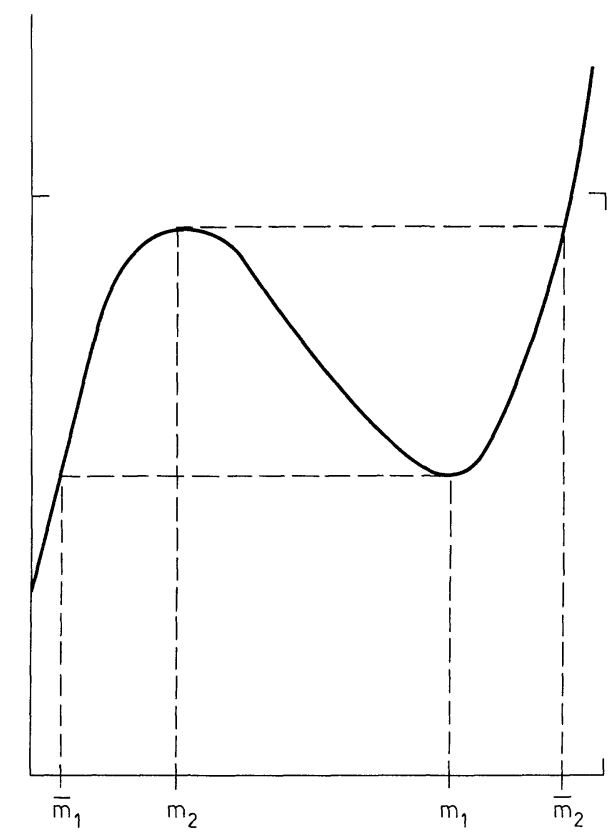

Fig. 2. The graph of the lift of a map in the class $A$

\footnotetext{
${ }^{1}$ Subsequent to the completion of this work I became aware of the paper of Kadanoff [20] in
} which he uses this construction to justify statements similar to portions of this section 


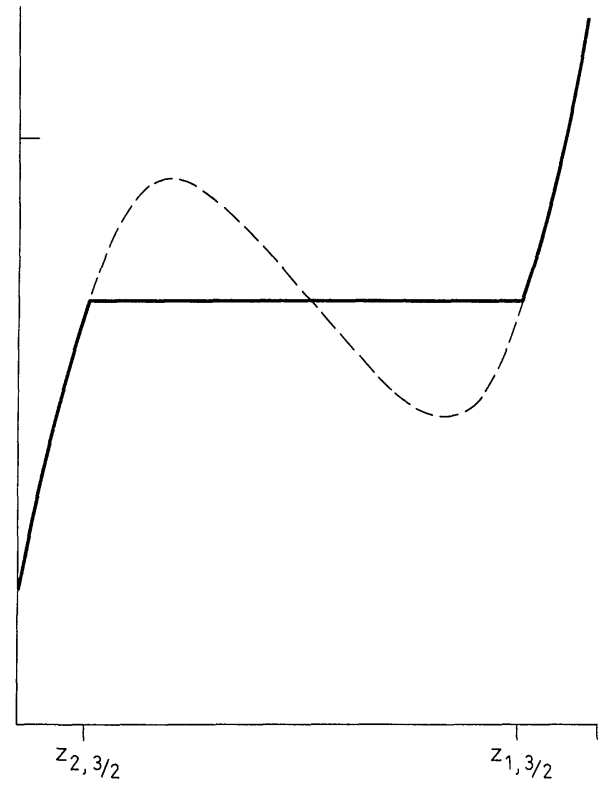

Fig. 3. The interpolated map $H_{3 / 2}$ in Hall's construction

(see Fig. 3). Note that $z_{11}=m_{1}$ and $z_{22}=m_{2}$. For convenience of notation we shall often let $\overline{m_{1}}=z_{2,1}$ and $\overline{m_{2}}=z_{1,2}$ (see Fig. 2).

For each $\mu \in[1,2]$ define $H_{\mu}:[0,1] \rightarrow \mathbb{R}$ via

$$
\begin{aligned}
H_{\mu}(x) & =G\left(m_{1}\right)+(\mu-1)\left(G\left(m_{2}\right)-G\left(m_{1}\right)\right) \text { for } x \text { in }\left[z_{2, \mu}, z_{1, \mu}\right] \\
& =G(x) \quad \text { otherwise. }
\end{aligned}
$$

Now extend $H_{\mu}$ to the real line subject to $H_{\mu}(x+1)=H_{\mu}(x)+1$ and let $h_{\mu}$ denote its projection onto a circle map. Note that $h_{\mu}$ is continuous and nondecreasing for all $\mu \in[1,2]$.

The next lemma examines maps that look like $h_{\mu}$. In its statement and throughout this work, $\alpha$ denotes an irrational number.

Lemma 2.1. Let $h: S^{1} \rightarrow S^{1}$ be nondecreasing, continuous and have precisely one interval $\left[z_{2}, z_{1}\right]$ with $h^{\prime}(x)=0$ for $x \in\left(z_{2}, z_{1}\right)$.

(a) If $\varrho(h)=\alpha \notin \mathbb{Q}$, then $o\left(z_{i}\right) \cap\left(z_{2}, z_{1}\right)=\emptyset$ for $i=1,2$.

(b) If $\varrho(h)=p / q$, then there is a $p / q$-periodic point $z$ with $o(z) \cap\left(z_{2}, z_{1}\right)=\emptyset$.

Proof. To prove (a), note that if for some $j, h^{j}\left(z_{i}\right) \in\left[z_{2}, z_{1}\right]$, then $h^{j+1}\left(z_{i}\right)=h\left(z_{i}\right)$, and so $\varrho\left(z_{i}\right)$ is rational, a contradiction.

To prove (b), first let $I=\left(z_{2}, z_{1}\right)$ and $y=h(I)$. By Lemma $1.2, h$ has a $p / q$ periodic point $y_{1}$. If $o\left(y_{1}\right) \cap I=\emptyset$, we are done, so assume that $o\left(y_{1}\right) \cap I \neq \emptyset$, and so $y$ is $p / q$-periodic. Let $y^{\prime}=h^{q-1}(y) \in I$ and $I_{-i}=h^{-i}(I)$.

We first show that the sets $I, I_{-1}, \ldots, I_{-(q-1)}$ are pairwise disjoint. Assume there is an $x \in I_{-i} \cap I_{-j}$ with $j>i$. Then $h^{i}(x) \in I$ and $h^{j}(x) \in I$, and so $h^{i+1}(x)$ $=h^{j+1}(x)=y$. Thus $h^{j-i}(y)=h^{j-i}\left(h^{i+1}(x)\right)=h^{j+1}(x)=y$, and $y$ is therefore periodic with period $j-i<q$, a contradiction. 
Now since the $I_{-i}$ are disjoint for $0 \leqq i \leqq q-1, h^{j}\left(I_{-i}\right) \cap I=\emptyset$ for all $j<i$, and so $h^{i} \mid I_{-i}$ is a homeomorphism onto $I$, so for each $i, I_{-i}$ is an open interval. And note that $h^{q}\left(I_{-i}\right)=h^{q-i-1}(h(I))=h^{-i}\left(h^{q-1}(y)\right)=h^{-i}\left(y^{\prime}\right) \in I_{-i}$, and so $h^{q}\left(I_{-i}\right) \in I_{-i}$ for all $0 \leqq i \leqq q-1$.

If we let $I_{-j}=\left(h^{-j}\left(z_{2}\right), h^{-j}\left(z_{1}\right)\right)$ be the next $I_{-i}$ to the right of $I=\left(z_{2}, z_{1}\right)$ (see Fig. 4) then using the fact that $h$ is nondecreasing,

$$
h^{q}\left(\left[z_{1}, h^{-j}\left(z_{2}\right)\right]\right) \supseteqq\left[z_{1}, h^{-j}\left(z_{2}\right)\right] .
$$

We therefore have a $p / q$-periodic point $z \in\left[z_{1}, h^{-j}\left(z_{2}\right)\right]$ and by construction, $0(z) \cap I=\emptyset$.

The next lemma uses Hall's construction to give a nice interpretation of the rotation interval of a map $g \in A$. Specifically, each number in the rotation interval of $g$ is the rotation number of one of the interpolated nondecreasing maps $h_{\mu}$.

Lemma 2.2. $g$ is a map in the class $A$ and for each $\mu \in[1,2], h_{\mu}$ is the interpolated map given above.

(a) $\varrho\left(h_{i}\right)=\varrho_{i}(g)$ for $i=1,2$.

(b) For each $r \in \varrho(g)$, there is a $\mu \in[1,2]$ with $r=\varrho\left(h_{\mu}\right)$.

Proof. $H_{1}$ is nondecreasing and $G \geqq H_{1}$, so by Lemma 1.6(c), $\varrho_{1}(G) \geqq \varrho\left(H_{1}\right)$. On the other hand, Lemma 2.1 yields a point $z$ with $o(z, G)=o\left(z, H_{1}\right)$, so $\varrho\left(H_{1}\right)=\varrho\left(z, H_{1}\right)$ $=\varrho(z, G) \geqq \varrho_{1}(G)$ which proves (a). Now by Theorem 1.3, $\varrho\left(H_{\mu}\right)$ is a continuous function of $\mu$, so part (a) with the intermediate value theorem proves (b).

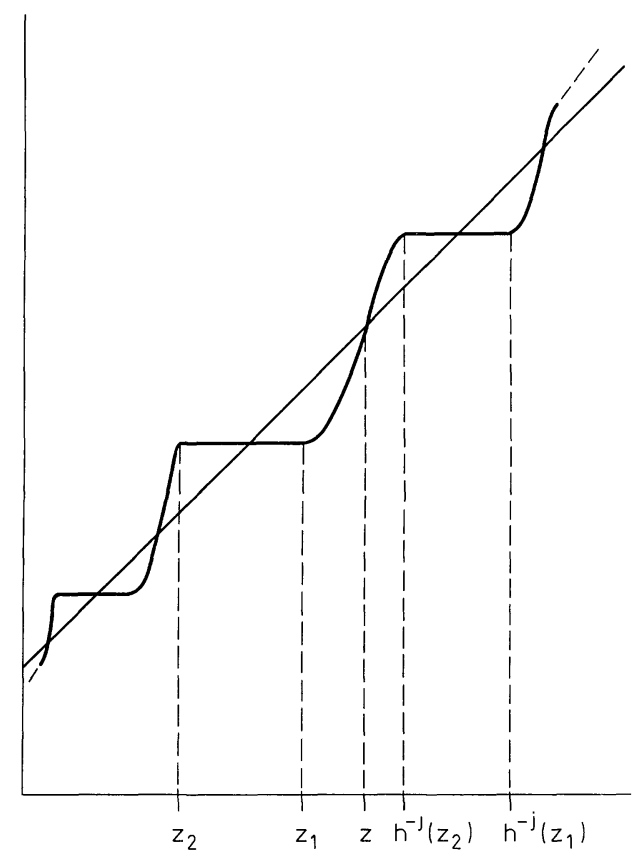

Fig. 4. The graph of $H^{q}$ in the proof of Lemma 2.1 
For $g$ in $A$, define $\Sigma(g)=\left\{x \in S^{1}: o(x, g) \cap\left(m_{2}, m_{1}\right)=\emptyset\right\}$. Note that

$$
\Sigma(g)=\bigcap_{i=0}^{\infty} g^{-i}\left(\left[m_{1}, m_{2}\right]\right)
$$

and is thus compact and invariant.

Proposition 2.3. $p / q$ and $\alpha$ are rational and irrational respectively, $g$ is in $A$.

(a) $p / q \in \varrho(g)$ if and only if there exists a $p / q$-periodic point $z \in \Sigma(g)$.

(b) $\alpha \in \varrho(g)$ if and only if there exists a $z \in \Sigma(g)$ with $\varrho(z)=\alpha$.

(c) For $i=1$ or $2, \varrho_{i}(g)=\alpha$ if and only if $\varrho\left(m_{i}\right)=\alpha$ and for $i=1, o\left(m_{1}\right)$ $\cap\left[\bar{m}_{1}, m_{1}\right)=\emptyset$ and for $i=2, o\left(m_{2}\right) \cap\left(m_{2}, \bar{m}_{2}\right]=\emptyset$. In particular, $\varrho_{i}(g)=\alpha$ implies that $m_{i} \in \Sigma(g)$.

Proof. We first note that the "if" portion of (a) is obvious. If $p / q \in \varrho(g)$, use Lemma 2.2 to find a $\mu$ with $\varrho\left(h_{\mu}\right)=p / q$. Then by Lemma 2.1(b) there is a $p / q$-periodic point $z$ with $o\left(z, h_{\mu}\right)=o(z, g)$ so $z \in \Sigma(g)$. The proof of (b) is similar. Now assume that $\varrho_{1}(g)=\alpha$. Then by Lemma 2.2(a), $\varrho\left(h_{1}\right)=\alpha$, and so by Lemma 2.1(a), $o\left(m_{1}\right) \cap\left[\bar{m}_{1}, m_{1}\right)=\emptyset$, and so $m_{1} \in \Sigma(f)$. For the converse, if $o\left(m_{1}\right)$ $\cap\left[\bar{m}_{1}, m_{1}\right)=\emptyset$, then $o\left(m_{1}, h_{1}\right)=o\left(m_{1}, g\right)$, and so $\alpha=\varrho\left(m_{1}, g\right)=\varrho\left(m_{1}, h_{1}\right)=\varrho\left(h_{1}\right)$ $=\varrho_{1}(g)$.

There are results of Bernhardt derived in [3] using kneading theory that can be shown to be equivalent to Proposition 2.3(a).

For a given map $g$ in $A$ and an $r$ in $\varrho(g)$, there are in general many, perhaps very complicated orbits $o(x)$ with $\varrho(x)=r$. Proposition 2.3 says that we can always find a particularly simple orbit $o(z)$ with $\varrho(z)=r$. In fact, by analyzing its proof one sees that $o(z)$ behaves like the orbit of a circle homeomorphism. Specifically, $o(z)$ is contained in the region where $g$ is increasing,

$$
\lim _{n \rightarrow \infty} \frac{G^{n}(z)-z}{n}
$$

exists and the order of the orbit of $z$ around the circle is the same as that of the orbit of a circle homeomorphism with rotation number $r$.

The next proposition will not be used in the sequel but we include it for its intrinsic interest.

Proposition 2.4. Given $a g \in A$ and $r \in \varrho(g)$, there exists a minimal set $\Lambda_{r} \subseteq \Sigma(g)$ with $\varrho(x)=r$ for all $x \in \Lambda_{r}$.

Proof. Use Lemma 2.2(b) to find a $\mu$ with $\varrho\left(h_{\mu}\right)=r$. Using Lemma 2.1,

$$
S^{1}-\bigcup_{i=0}^{\infty} h_{\mu}^{-i}\left(\left(z_{2 \mu}, z_{1 \mu}\right)\right)
$$

is a nonempty, closed, $h_{\mu}$-invariant set which thus contains a minimal set $\Lambda_{r}$. Since $\Lambda_{r} \subseteq S^{1}-\left(z_{2 \mu}, z_{1 \mu}\right)$, for all $x \in \Lambda_{r}, o\left(x, h_{\mu}\right)^{\cdot}=o(x, g)$, and thus $\varrho(x, g)=\varrho\left(x, h_{\mu}\right)=r$ and $h_{\mu}$ and $g$ are identical when restricted to $\Lambda_{r}$.

When $r=p / q$, the set $\Lambda_{p / q}$ can be just a $p / q$-periodic orbit. When $r=\alpha$ an irrational, however, $\Lambda_{\alpha}$ is a minimal set similar to those in Denjoy counter- 
examples. It can be viewed as the one dimensional analog of an Aubry-Mather invariant Cantor set.

We conclude this chapter by studying the behavior of the rotation interval in a particularly simple type of one parameter family. Let $g$ be a degree one circle map and $G$ its lift with $G(0) \in[0,1)$. For each $w \in \mathbb{R}$ define $G_{w}=G+w$, and let $g_{w}$ be the circle map which is the projection of $G_{w}$.

When $g$ is a circle homeomorphism which satisfies $G_{w}^{q} \neq R_{p}$ whenever $\varrho\left(g_{w}\right)$ $=p / q, \varrho(w)=\varrho\left(g_{w}\right)$ is a continuous and nondecreasing function of $w$ with $\varrho^{-1}(p / q)$ a nontrivial closed interval and $\varrho^{-1}(\alpha)$ is a point [16].

The next lemma states that $\varrho_{1}(w)=\varrho_{1}\left(G_{w}\right)$ and $\varrho_{2}(w)=\varrho_{2}\left(G_{w}\right)$ have these same properties when $g \in A$. The main element in the proof is that $\varrho\left(H_{i}\right)=\varrho_{i}(G)$ where for $i=1,2$ the $H_{i}$ are the extreme interpolated maps in Hall's construction (see Lemma 2.2). Thus $\varrho_{i}\left(G_{w}\right)$ is controlled by the one parameter family of nondecreasing maps $H_{i, w}=H_{i}+w$ and we know that nondecreasing maps behave like homeomorphisms with regard to their rotation numbers.

Lemma 2.5. Let $g \in A$ and $G_{w}$ and $H_{i, w}$ be as defined above. For $i=1,2, \varrho_{i}: \mathbb{R} \rightarrow \mathbb{R}$ is continuous, nondecreasing and onto. For irrational $\alpha, \varrho_{i}^{-1}(\alpha)$ is a point while $\varrho_{i}^{-1}(p / q)$ is a nontrivial closed interval. Fix $p / q$ and let $\varrho_{i}^{-1}(p / q)=\left[\Psi_{1}, \Phi_{1}\right]$ and $\varrho_{2}^{-1}(p / q)=\left[\Phi_{2}, \Psi_{2}\right]$.

(a) $H_{i, w}^{q} \leqq R_{p}$ with equality attained iff $w=\Psi_{1}$, if $i=1$ and $w=\Phi_{2}$ if $i=2$.

(b) $H_{i, w}^{q} \geqq R_{p}$ with equality attained iff $w=\Phi_{1}$, if $i=1 i=1$ and $w=\Psi_{2}$ for $i=2$.

(c) There exist $z_{1}$ and $z_{2}$ with $H_{i, w}^{q}\left(z_{1}\right)>z_{1}+p$ and $H_{i, w}^{q}\left(z_{2}\right)<z_{2}+p$ iff $w \in\left(\Psi_{1}, \Phi_{1}\right)$ for $i=1$ and $w \in\left(\Phi_{2}, \Psi_{2}\right)$ for $i=2$.

Proof. Continuity of $\varrho_{1}$ follows from Theorem 1.3. By Lemma 2.2(a), $\varrho_{1}\left(g_{w}\right)$ $=\varrho\left(h_{1, w}\right)$. If $w_{1}>w_{2}$, then $H_{1, w_{2}}>H_{1, w_{2}}$, and so by Lemma 1.6, $\varrho\left(h_{1, w_{1}}\right) \geqq \varrho\left(h_{1, w_{2}}\right)$, so $\varrho_{1}$ is nondecreasing. $\varrho_{1}$ is onto since one may easily adjust $w$ so that $\varrho\left(H_{1, w}\right)=n$ for any integer $n$. We can therefore conclude that for any real number $r, p_{1}^{-1}(r)$ is either a point or a nontrivial closed interval.

As already noted, $w_{1}>w_{2}$ implies $H_{1, w_{1}}>H_{1, w_{2}}$ and so by Lemma 1.6, $\varrho\left(H_{1, w_{1}}\right)>\varrho\left(H_{1, w_{2}}\right)$ if either of them is irrational. This shows that $\varrho_{1}^{-1}(\alpha)$ is a point.

Now choose $\beta \in B d\left(\varrho_{1}^{-1}(p / q)\right)$. Since $\varrho_{1}\left(g_{w}\right)=\varrho\left(h_{1, w}\right)$ it's clear that $h_{1, \beta}$ is in $B d\left(E_{p / q}\right)$. Thus by Lemma 1.4 either

(1) $H_{1, \beta}^{q} \geqq R_{p}$ with equality attained or

(2) $H_{2, \beta}^{q} \leqq R_{p}$ with equality attained.

Now if $H_{1, \beta}^{q}=R_{p}$, then $h_{1, \beta}^{q}=\mathrm{Id}$, and so $h_{1, \beta}$ is injective, a contradiction. Thus if we assume (1) holds, we may find an $\varepsilon>0$ so that there is a point $z$ with $H_{1, \beta-\varepsilon}^{q}(z)$ $=z+p$ or $p / q=\varrho\left(H_{1, \beta-\varepsilon}\right)=\varrho_{1}\left(G_{\beta-\varepsilon}\right)$. One handles the case where $(2)$ holds similarly showing that $\varrho_{1}^{-1}(p / q)$ is a nontrivial closed interval. The rest of the lemma follows easily from Lemmas 1.4 and 1.6.

We conclude this section by noting that $H_{1, w}^{q} \geqq R_{p}$ (respectively, $H_{2, w}^{q} \leqq R_{p}$ ) with equality attained if and only if $G_{w}^{q} \geqq R_{p}\left(G_{w}^{q} \leqq R_{p}\right)$ with equality attained. The proof is easy. This gives an alternative characterization of $\Phi_{1}$ and $\Phi_{2} . w=\Phi_{1}$ iff $G_{w}^{q} \geqq R_{p}$ with equality attained and $w=\Phi_{2}$ iff $G_{w}^{q} \leqq R_{p}$ with equality attained. We also note that many of the results of this section may be generalized to degree one circle maps with a finite number of turning points. The proofs utilize the obvious generalization of Hall's construction. 


\section{Construction of the Bifurcation Diagram}

We have now developed enough machinery to begin our study of two parameter families. The families we shall study are of the form $F_{b, w}(x)=F(x, b, w)=x+w$ $+b p(x)$ for $x, w$ in $\mathbb{R}$ and $b$ in $[0, \infty)$. In addition, $p: \mathbb{R} \rightarrow \mathbb{R}$ is continuous and periodic, $(p(x+1)=p(x))$. This forces $F_{b, w}(x+1)=F_{b, w}(x)+1$, so that $F_{b, w}$ is the lift of a continuous degree one circle map which we denote $f_{b, w}$. In constructing the bifurcation diagram of the family it will usually be more convenient to work with $F_{b, w}$.

Since we want the family to look qualitatively like the socalled canonical family, $p(x)=\sin (2 \pi x) / 2 \pi$, we also require that $p$ is such that the following hypotheses are satisfied.

Hypothesis $0 . \operatorname{Max}(p(x))=1 / 2 \pi=-\min (p(x))$ and $p(0)=0=p(1)$.

Hypothesis 1 . For all $b>1, f_{b, w}$ is in $A$.

Hypothesis 2. For $b<1, f_{b, w}$ is a homeomorphism and when $b=1, f_{b, w}$ is nondecreasing.

Hypothesis 3. For $0<b<1, F_{b, w}^{q} \neq R_{p}$ whenever $\varrho\left(F_{b, w}\right)=p / q$.

It is fairly easy to find hypotheses on $p$ which force Hypotheses 1 and 2 to be satisfied. We shall give several in Sect. 4 which apply when $p$ is $C^{1}$. Herman [16] has shown that Hypothesis 3 holds for a generic function in the space $C^{r}\left(S^{1}, \mathbb{R}\right)$ and is satisfied when $p$ is the restriction of a complex entire function to the real line. In particular, it is true for the canonical family.

Another example to keep in mind for $p$ is the piecewise linear map shown in Fig. 5. We note that our hypotheses do not require $p$ to display the symmetry these two examples have. Specifically, it is not necessary that $p(x)=-p(1-x)$. Also, the requirement in Hypothesis 0 that $\max (p(x))=-\min (p(x))$ could be dispensed with but it would unnecessarily complicate the exposition. Finally, the choice of $1 / 2 \pi$ for the maximum of $p(x)$ is arbitrary and was chosen simply so that our results would apply to the canonical family directly.

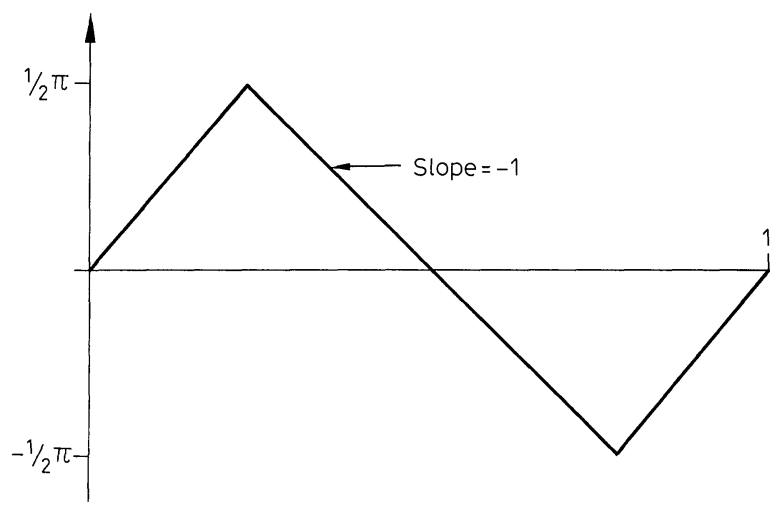

Fig. 5. A piecewise linear example for $p(x)$ 
Now fix a $p(x)$ that satisfies all the hypotheses above. To investigate how $\varrho\left(f_{b, w}\right)$ varies, we will examine the $(b, w)$-bifurcation diagram in terms of sets $T_{r}=\left\{(b, w): r \in \varrho\left(F_{b, w}\right)\right\}$ and for $i=1,2, S_{i, r}=\left\{(b, w): r=\varrho_{i}\left(F_{b, w}\right)\right\}$.

For a fixed $b>1, f_{b, 0}$ is in the class $A$, and so applying Lemma 2.5 to the one parameter family $F_{b, 0}+w=F_{b, w}$, we have for each rational, $p / q$, numbers $\Psi_{1, p / q}$ and $\Phi_{1, p / q}$ with $\varrho_{1}\left(F_{b, w}\right)=p / q$ if and only if $w \in\left[\Psi_{1, p / q}, \Phi_{1, p / q}\right]$. Thus including the dependence of $\Phi_{1, p / q}$ and $\Psi_{1, p / q}$ on $b$, we have functions $\Phi_{1, p / q}, \Psi_{1, p / q}:(1, \infty) \rightarrow \mathbb{R}$ with

$$
S_{1, p / q}=\left\{(b, w): \Psi_{1, p / q}(b) \leqq w \leqq \Phi_{1, p / q}(b)\right\} .
$$

Similarly, there are functions $\Phi_{2, p / q}$ and $\Psi_{2, p / 2}$ with

$$
S_{2, p / q}=\left\{(b, w): \Phi_{2, p / q}(b) \leqq w \leqq \Psi_{2, p / q}(b)\right\} .
$$

Now if $\alpha$ is irrational, again using Lemma 2.5 , let $\Phi_{1, \alpha}$ be the unique value of $w$ with $\varrho_{1}\left(F_{b, w}\right)=\alpha$. Then

$$
S_{1, \alpha}=\left\{(b, w): w=\Phi_{1, \alpha}(b)\right\} .
$$

Similarly, there is a function $\Phi_{2, \alpha}:(1, \infty) \rightarrow \mathbb{R}$ with

$$
S_{2, \alpha}=\left\{(b, w): w=\Phi_{2, \alpha}(b)\right\} .
$$

The main theorem of this section states that all the functions just defined are Lipschitz with constant $K=1 / 2 \pi$. Also,

$$
T_{r}=\left\{(b, w): \Phi_{2, r}(b) \leqq w \leqq \Phi_{1, r}(b)\right\},
$$

which means that the boundaries of the $T_{r}$ are graphs of continuous functions.

As mentioned in the introduction, the structure of the $T_{r}$ when $b<1$ is given in Herman [15]. Using a lemma of Herman (which requires Hypothesis 3) one may define functions on $[0,1]$ which make statements (1)-(4) above true in the region $b<1$ (see remarks before Lemma 2.5, the case $b=1$ requires a trivial extension of Herman's lemma). However, since $f_{b, w}$ is a homeomorphism when $b \leqq 1, \varrho_{1}\left(f_{b, w}\right)$ $=\varrho_{2}\left(f_{b, w}\right)$ and so for all $r, S_{1, r}=S_{2, r}$. Thus for rational $p / q, \Psi_{2, p / q}=\Phi_{1, p / q}$ and $\Psi_{1, p / q}=\Phi_{2, p / q}$, while for irrational $\alpha, \Phi_{1, \alpha}=\Phi_{2, \alpha}$.

The prototype for $T_{p / q}$ for a rational $p / q$ is $T_{n}$, where $n$ is an integer. One may easily compute, using results from Sect. 2 , that $\Phi_{1, n}(b)=n+b / 2 \pi, \Phi_{2, n}(b)=n$ $-b / 2 \pi$, and

$$
T_{n}=\{(b, w): n-b / 2 \pi \leqq w \leqq n+b / 2 \pi\} .
$$

We also note that a more detailed study of the structure of the $T_{r}$ near $b=0$ and its dependence on properties of the function $p$ is given in Hall [14].

We now state the main theorem of this section which gives the structure of the $T_{r}$ (see Fig. 6).

Theorem 3.1. $r$ is an arbitrary real number, $\alpha$ is irrational and $p / q$ is rational.

(a) For $i=1$ or 2 , the functions $\Phi_{i, r}$ and $\Psi_{i, p / q}$ defined above are Lipschitz with constant $K=1 / 2 \pi$.

(b) $\Phi_{2, r} \leqq \Phi_{1, r}, \Phi_{2, p / q} \leqq \Psi_{1, p / q}$, and $\Psi_{2, p / q} \leqq \Phi_{1, p / q}$. When $b>0, \Psi_{1, p / q}<\Phi_{1, p / q}$, and $\Phi_{2, p / q}<\Psi_{2, p / q}$.

$$
T_{r}=\left\{(b, w): \Phi_{2, r}(b) \leqq w \leqq \Phi_{1, r}(b)\right\}
$$




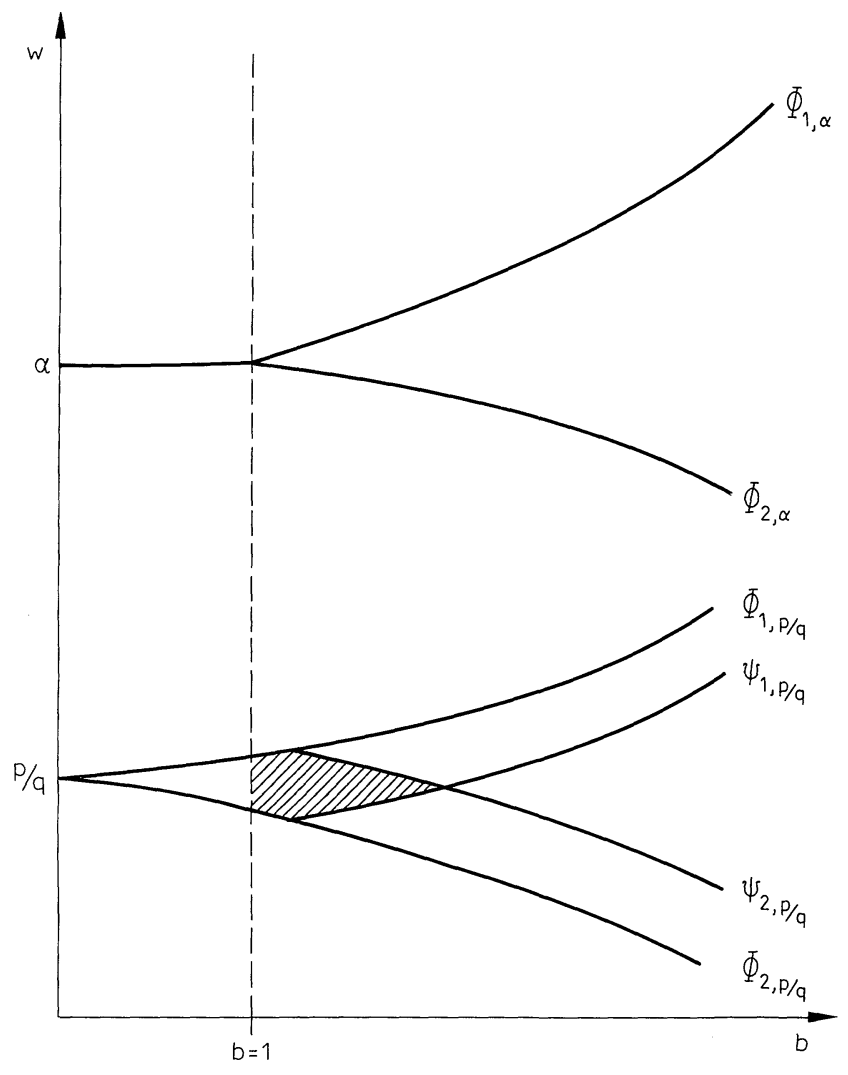

Fig. 6. Idealized picture of the $T_{r}$

Before proving the main theorem, we state a simple lemma which describes the convergence of the functions defined above. It is an easy consequence of Lemma 2.5. The notation $f_{r} \searrow f$ means that the $f_{r}$ converge to $f$ and for $r_{1}>r_{2}$, $f_{r_{1}}>f_{r_{2}}$. Lemma 3.2 is illustrated in Fig. 7.

Lemma 3.2. $p / q, \alpha$, and $r$ are rational, irrational and arbitrary real numbers respectively.

(a) For $r>p / q, \Phi_{1, r} \searrow \Phi_{1, p / q}$, and $\Phi_{2, r} \searrow \Psi_{2, p / q}$.

(b) For $r<p / q, \Phi_{1, r} \nearrow \Psi_{1, p / q}$, and $\Phi_{2, r} \nearrow \Phi_{2, p / q}$.

(c) For $r>\alpha$ and $i=1,2, \Phi_{i, r} \searrow \Phi_{i, \alpha}$.

(d) For $r<\alpha$ and $i=1,2, \Phi_{i, r} \nearrow \Phi_{i, \alpha}$.

Given the map $F_{b, w}$ with $b>1$, the maps $H_{1, b, w}$ and $H_{2, b, w}$ are the extreme interpolated maps from the construction in Sect. 2. In the proof of Theorem 3.1, it will be convenient to have these maps defined when $b \leqq 1$ as $H_{1, b, w}=H_{2, b, w}=F_{b, w}$.

Proof of Theorem 3.1. (a) Fix a $p / q$. We shall prove the result for $\Phi_{1, p / q}$ (which we shall denote as $\Phi$ ). 


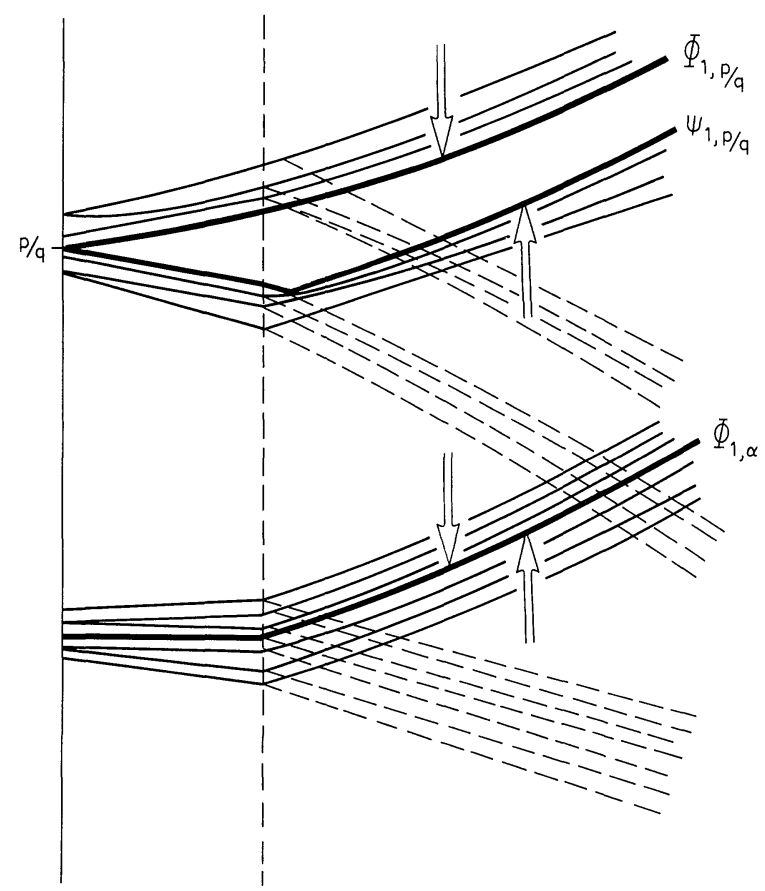

Fig. 7. Illustration of the convergence in Lemma 3.2

If we assume there is a $b_{0}$ where $\Phi$ is not Lipschitz with constant $K=1 / 2 \pi$, then there is a $b_{1}$ so that the points $\left(b_{0}, \Phi\left(b_{0}\right)\right)$ and $\left(b_{1}, \Phi\left(b_{1}\right)\right)$ lie on a line with slope $\bar{m}>1 / 2 \pi$. [We may assume without loss of generality that $\Phi\left(b_{1}\right)>\Phi\left(b_{0}\right)$.] Thus, there is a $\bar{c}$ with $\bar{m} b_{0}+\bar{c}=\Phi\left(b_{0}\right)$ and $\bar{m} b_{1}+\bar{c}=\Phi\left(b_{1}\right)$. Letting $m=1 / \bar{m}$ and $c=-\bar{c} / \bar{m}$, we have $m \Phi\left(b_{0}\right)+c=b_{0}$ and $m \Phi\left(b_{1}\right)+c=b_{1}$. Now since $|m|<2 \pi$ and $|p(x)| \leqq 1 / 2 \pi$, certainly $m p(x)>-1$, and so

$$
m p(x)>\left(\Phi\left(b_{1}\right)-\Phi\left(b_{0}\right)\right) /\left(\Phi\left(b_{0}\right)-\Phi\left(b_{1}\right)\right) .
$$

We therefore have that

$$
x+\Phi\left(b_{0}\right)+\left(m \Phi\left(b_{0}\right)+c\right) p(x)<x+\Phi\left(b_{1}\right)+\left(m \Phi\left(b_{1}\right)+c\right) p(x)
$$

which says that $\left.F_{b_{0}, \Phi\left(b_{0}\right)}<F_{b_{1}, \Phi\left(b_{1}\right)}\right)$ and so $H_{1, b_{0}, \Phi\left(b_{0}\right)}<H_{1, b_{1}, \Phi\left(b_{1}\right)}$. We therefore have $H_{I, b_{0}, \Phi,\left(b_{0}\right)}^{q}<H_{I, b_{1}, \Phi\left(b_{1}\right)}^{q}$ by Lemma 1.6(b). But by definition, $H_{1, b_{1}, \Phi\left(b_{1}\right)}^{q} \leqq R_{p}$, and so $H_{1, b_{0}, \Phi\left(b_{0}\right)}^{q}<R_{p}$. Recalling that $\Phi\left(b_{0}\right)=\Phi_{1, p / q}\left(b_{0}\right)$, we have $p / q$ $=\varrho\left(H_{1, b_{0}, \Phi\left(b_{0}\right)}\right)<p / q$, a contradiction.

The proof for $\Phi_{2, p / q}$ and the $\Psi_{i, p / q}$ are similar. If $r$ is irrational, one uses Lemma 3.2.

(b) We first show that $\Psi_{2, p / q} \leqq \Phi_{1, p / q}$. To simplify notation, drop the dependence on $p / q$ and let $\varrho\left(F_{b, w}\right)=\varrho(b, w)$. Assume to the contrary that $\Phi_{1}<\Psi_{2}$. Since $\Phi_{1}(b)$ is the maximum value of $w$ with $\varrho_{1}(b, w)=p / q$, using the monotonicity of $\varrho_{1}$ we have

$$
p / q=\varrho_{1}\left(b, \Phi_{1}(b)\right)<\varrho_{1}\left(b, \Psi_{2}(b)\right) \leqq \varrho_{2}\left(b, \Psi_{2}(b)\right)=p / q,
$$

a contradiction. 
The proof of $\Phi_{2, p / q} \leqq \Psi_{1, p / q}$ is similar. The proof that $\Phi_{2, \alpha} \leqq \Phi_{1, \alpha}$ is also essentially the same as above. The last two inequalities in part (b) are direct consequences of Lemma 2.5. Finally, $\Phi_{2, p / q} \leqq \Psi_{1, p / q}<\Phi_{1, p / q}$ completing the proof of (b).

(c) For $i=1,2$, let $\Phi_{i, r}=\Phi_{i}$. Choose a $(b, w)$ with $\Phi_{2}(b) \leqq w \leqq \Phi_{1}(b) . \Phi_{1}(b) \geqq w$ implies that $\alpha=\varrho_{1}\left(b, \Phi_{1}(b)\right) \geqq \varrho_{1}(b, w)$. Similarly, $\Phi_{2}(b) \leqq w$ implies that $\alpha \leqq \varrho_{2}(\mathrm{~b}, \mathrm{w})$, so $\alpha \in \varrho\left(f_{b, w}\right)$, ie. $(b, w) \in T_{\alpha}$.

Now since $\Phi_{1}(b)$ is the maximum $w$ (if $r$ is irrational, it is the unique $w$ ) with $\varrho_{1}(b, w)=\alpha$, we have $w>\Phi_{1}(b)$ implies that $\varrho_{1}(b, w)>\alpha$, so that $\alpha \notin \varrho\left(f_{b, w}\right)$ or $(b, w) \notin T_{\alpha}$. Similarly, $w<\Phi_{2}(b)$ also implies $(b, w) \notin T_{\alpha}$.

If we let $\Phi_{1}$ denote $\Phi_{1, p / q}$, then recall that $\left(b, \Phi_{1}(b)\right)$ [respectively, $\left.\left(b, \Phi_{2}(b)\right)\right]$ is characterized by $F_{b, \Phi_{1}(b)}^{q} \geqq R_{p}\left(F_{b, \Phi_{2}(b)}^{q} \leqq R_{p}\right)$ with equality attained. Theorem 3.1 thus says that when $(b, w)$ is on the boundary of $T_{p / q}, f_{b, w}$ has a $p / q$-saddle node.

We also note that since all the functions in Lemma 3.2 are continuous, Dini's Theorem says that the convergence in Lemma 3.2 is uniform on compact sets. This gives us another way of looking at the contents of Lemma 3.2. Consider the collection $B$ of all the $\Phi_{1, r}$ and $\Psi_{1, p / q}$, for $r$ and $p / q$ in some closed interval, as a subset of the function space $C([0, \infty), \mathbb{R})$. If we put the compact open topology on the function space, then $B$ is a Cantor set. Similar comments hold for the union of the $\Phi_{2, r}$ and $\Psi_{2, p / q}$.

The next proposition states that everything one expects to cross actually does so. The various cases are illustrated in Fig. 8.

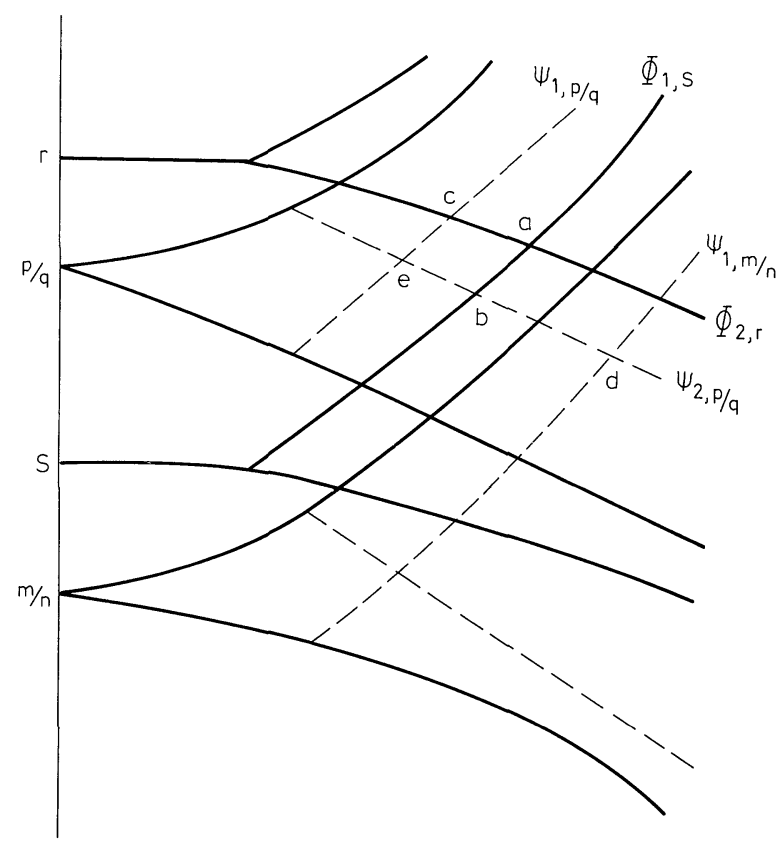

Fig. 8. Illustration of the various cases in Proposition 3.3. 
Proposition 3.3. $r$ and $s$ are arbitrary real numbers and $p / q$ and $m / n$ are rational with $r>p / q>s>m / n$. In each of the following cases there exists a $b>1$ such that the stated equality holds.

$$
\begin{gathered}
\Phi_{2, r}(b)=\Phi_{1, s}(b), \\
\Psi_{2, p / q}(b)=\Phi_{1, s}(b), \\
\Psi_{1, p / q}(b)=\Phi_{2, r}(b), \\
\Psi_{1, m / n}(b)=\Psi_{2, p / q}(b), \\
\Psi_{1, p / q}(b)=\Psi_{2, p / q}(b) .
\end{gathered}
$$

Proof. We prove (a), the rest are similar. First note that since $r>s, \Phi_{2, r}(0)>\Phi_{1, s}(0)$. Now choose integers $k$ and $l$ with $k>r>s>l$. Using the remark above the statement of Theorem 3.1 and Lemma 3.2, we have for $b>\pi(k-l), \Phi_{1, s}>\Phi_{1, l}$ $>\Phi_{2, k}>\Phi_{2, r}$, which using continuity, gives us the point needed for (a).

The results of this chapter thus far show that, to a large extent, the actual bifurcation diagram looks (topologically at least) like the idealized drawings we have presented in Figs. 6, 7 and 8. They do not, however, rule out the possibility of multiple intersections of the various curves. Proposition 3.3(e) implies the existence of the shaded region shown in Fig. 6 where $b>1$, but $\varrho\left(f_{b, w}\right)=p / q$. The dynamics of such $f_{b, w}$ could be quite complex, but all orbits are rotating at the same asymptotic speed.

Using an argument as in the proof of Proposition 3.3, it is easy to see that for $b>\pi, \Phi_{1, p / q}>\Psi_{2, p / q}$, and $\Phi_{1, \alpha}>\Phi_{2, \alpha}$. Since for $b<1$, both of these inequalities are equalities, one would like to know where the curves branch apart. The next proposition states that under additional differentiability assumptions, this branching takes place as shown in Fig. 6 , i.e. $\Phi_{1, p / q}$ splits from $\Psi_{2, p / q}$ in the region $b>1$ and $\Phi_{1, \alpha}$ and $\Phi_{2, \alpha}$ split on the line $b=1$. The proof of the second assertion (which is equivalent to saying that $T_{\alpha}$ opens out into a tongue at the line $b=1$ ) is essentially contained in the following lemma due to Block and Franke [5].

Lemma 3.4 (Block and Franke). Let $f: S^{1} \rightarrow S^{1}$ be $C^{1}$ with $f^{\prime}$ of bounded variation. If $\left\{x: f^{\prime}(x)=0\right\}$ is nonempty and finite and $f^{\prime}$ changes sign at each critical point, then $f$ has a periodic point.

Proposition 3.5. $\alpha$ is irrational, $p / q$ is rational.

(a) If $p(x)$ is $C^{2}$, then for $b>1, \Phi_{1, \alpha}>\Phi_{2, \alpha}$

(b) If $p(x)$ is $C^{1}$, then given a $p / q$, there exists $b_{0}>1$ so that $\Psi_{1, p / q}(b)=\Phi_{2, p / q}(b)$ and $\Phi_{1, p / q}(b)=\Psi_{2, p / q}(b)$ for $0 \leqq b \leqq b_{0}$.

Proof. (a) If for some $b_{1}>1, \Phi_{1, \alpha}\left(b_{1}\right)=\Phi_{2, \alpha}\left(b_{1}\right)=w_{1}$, then $\varrho\left(f_{b_{1}, w_{1}}\right)=\alpha$ and thus $f_{b_{1}, w_{1}}$ has no periodic points. We shall show that the maximum and minimum $\left(m_{2}\right.$ and $m_{1}$ ) are the only points where $f_{b, w}^{\prime}$ vanishes in a $C^{2}$ family of the type we are considering. This will complete the proof using Lemma 3.4.

Denote $f_{b_{1}, w}$ by $f$. Assume there exists a third point $z$ with $0=f^{\prime}(z)=1+b p^{\prime}(z)$, then $p^{\prime}(z)=p^{\prime}\left(m_{1}\right)=p^{\prime}\left(m_{2}\right)=-1 / b$. Let us assume that $z$ is in $\left(m_{2}, m_{1}\right)$. Since $f$ is decreasing on $\left(m_{2}, m_{1}\right)$ (by Hypothesis 1$), p^{\prime}(x) \leqq-1 / b$ for all $x$ in $\left(m_{2}, m_{1}\right)$. Now 
$p^{\prime}(x) \equiv-1 / b$ for $x \in\left(m_{2}, m_{1}\right)$ is impossible, so there must exist $x_{1} \in\left(m_{1}, z\right)$ and $x_{2} \in\left(z, m_{2}\right)$ with $p^{\prime}\left(x_{i}\right)<-1 / b$. Using the continuity of $p^{\prime}$, one may choose a $b_{2}$ with $1<b_{2}<b_{1}$ so that $f_{b_{2}, w_{1}}$ is decreasing in neighborhoods of $x_{1}$ and $x_{2}$ and increasing in neighborhoods of $m_{1}, z$ and $m_{2}$. This implies that $f_{b_{2}, w}$ is not in the class $A$, a contradiction. The case $z \in\left(m_{1}, m_{2}\right)$ is treated similarly.

(b) Since $p / q$ is fixed, we do not include dependence on it in our notation. We shall show that $\Phi_{1}(b)=\Psi_{2}(b)$, the other equality is similar.

By Lemma 2.5 and the comments after it, $\Phi_{1}(b)$ [respectively $\left.\Psi_{2}(b)\right]$ is the unique $w$ with $F_{b, w}^{q} \geqq R_{p}\left(H_{2, b, w}^{q} \geqq R_{p}\right)$ with equality attained. Now since $H_{2, b, \Phi_{1}(b)}^{q}$ $\geqq F_{b, \Phi_{1}(b)}^{q} R_{p}$, if there is a $b_{0}>1$ such that for all $b<b_{0}$, the point $y(b)$ which satisfies $F_{b, \Phi_{1}(b)}^{q}(y(b))=y(b)+p$ also satisfies

$$
o\left(y(b), H_{2, b, \Phi_{1}(b)}\right)=o\left(y(b), F_{b, \Phi_{1}(b)}\right),
$$

then $H_{2, b, \Phi_{1}(b)}^{q}(y(b))=y(b)+p$, and so $\Psi_{2}(b)=\Phi_{1}(b)$, and we are done.

To get a contradiction, assume that such a $b_{0}$ does not exist. We may then find a sequence $b_{n} \rightarrow 1$ so that for all $n$,

$$
o\left(y\left(b_{n}\right), H_{2, b_{n}, \Phi_{1}\left(b_{n}\right)}\right) \neq o\left(y\left(b_{n}\right), F_{b_{n}, \Phi_{1}\left(b_{n}\right)}\right) .
$$

For this to happen, some element of the orbit of $y\left(b_{n}\right)$ under $F_{b_{n}, \Phi_{1}\left(b_{n}\right)}$ must land in $\left(m_{2}\left(b_{n}\right), \bar{m}_{2}\left(b_{n}\right)\right)$. Call this element of the orbit $y_{n}^{\prime}$. But as $n \rightarrow \infty, b_{n} \rightarrow 1$ and so $\bar{m}_{2}\left(b_{n}\right)$ $\rightarrow m_{2}(1)$, thus $y_{n}^{\prime} \rightarrow m_{2}(1)$ and therefore

$$
D F_{b_{n}, \Phi_{1}\left(b_{n}\right)}^{q}\left(y_{n}\right) \rightarrow D F_{1, \Phi_{1}(1)}^{q}\left(m_{2}(1)\right)=0
$$

(note: $D F^{q}$ means $\partial F^{q} / \partial x$ ). But $y_{n}^{\prime}$ was chosen so that $D F_{b_{n}, \Phi_{1}\left(b_{n}\right)}^{q}\left(y_{n}^{\prime}\right)=1$ for all $n$, a contradiction.

The smoothness assumption is necessary in part (b) as one may show that $\Psi_{1, p / q}$ splits off from $\Phi_{2, p / q}$ at the line $b=1$ for the piecewise linear example shown in Fig. 5. We also note that Block and Franke [5] alter a $C^{1}$ circle diffeomorphism which is a Denjoy counterexample and obtain a $C^{1}$ map in the class $A$ with no periodic points. It appears likely, however, that such maps do not occur in families of the type we are considering, and thus Proposition 3.5(a) would hold without a smoothness assumption.

A final remark: If we let $b_{0}(p / q)$ denote the $b_{0}$ found for $p / q$ in (b) above and we choose a sequence of rationals $p_{n} / q_{n} \rightarrow \alpha$, an irrational, then Lemma 3.2 with Proposition 3.5 implies that $b_{0}\left(p_{n} / q_{n}\right) \rightarrow 0$.

\section{Stable Periodic Orbits}

In this section we describe some additional structure of the bifurcation diagram relating to the existence of stable periodic orbits. We shall assume that our family

$$
F(x, b, w)=F_{b, w}(x)=x+w+b p(x)
$$

is $C^{r}$ with $r \geqq 1$. Under this assumption we can give some simple hypotheses which imply Hypothesis 1 and 2 of Sect. 3. 
Hypothesis 4. $p^{\prime}(0)>0$ and there exists $m_{0} \in(0,1)$ with $p^{\prime}\left(m_{0}\right)=-1$. If $p$ is $C^{1}$ (respectively, $\left.C^{2}\right), p^{\prime}$ is strictly decreasing $\left(p^{\prime \prime}<0\right)$ on $\left(0, m_{0}\right)$ and strictly increasing $\left(p^{\prime \prime}>0\right)$ on $\left(m_{0}, 1\right)$.

Throughout the remainder of this paper we shall assume that $p(x)$ satisfies Hypotheses 0 to 4 . We note that this implies that $m_{1}$ and $m_{2}$ are continuous functions of $b$ which we denote $m_{1}(b)$ and $m_{2}(b)$ and that $m_{1}(1)=m_{2}(1)=m_{0}$.

We recall some standard definitions. A point $z$ is a stable fixed point for a map $f: S^{1} \rightarrow S^{1}$ if $f(z)=z$ and there is an interval $N$ containing $z$ with $f^{n}(x) \rightarrow z$ as $n \rightarrow \infty$ for all $x$ in $N$. The condition $|D f(z)|<1$ is sufficient to make $z$ a stable fixed point. $z$ is a member of a stable $p / q$-periodic orbit if $z$ is $p / q$-periodic and is a stable fixed point for $f^{q}$. If in addition, the orbit of $z$ contains a critical point of $f$, then $z$ belongs to a superstable $p / q$-periodic orbit.

As we mentioned in the previous section, the set $T_{0}$ is the prototype for $T_{p / q}$ and much of its structure can be computed explicitly. For example, we showed that its boundaries are the graphs of the functions $\Phi_{1,0}(b)=b / 2 \pi$ and $\Phi_{2,0}(b)=-b / 2 \pi$. If we restrict ourselves to the canonical family, $F_{b, w}(x)=x+w+(b / 2 \pi) \sin (2 \pi x)$, one can also compute that a critical point of $F_{b, w}$ is a fixed point precisely when $(b, w)$ is on the hyperbola $b^{2}-4 \pi^{2} w^{2}=1$. For $(b, w)$ in the region between the hyperbola and the boundary of $T_{0}, F_{b, w}$ has a stable fixed point. The situation is illustrated in Fig. 9. The theorem we prove in this section says that this same basic structure holds in $T_{p / q}$.

The following fact is essentially contained in Collet and Eckmann [7] and will be used in the proof: If $f:[x, y] \rightarrow[x, y]$ is continuous and orientation preserving,
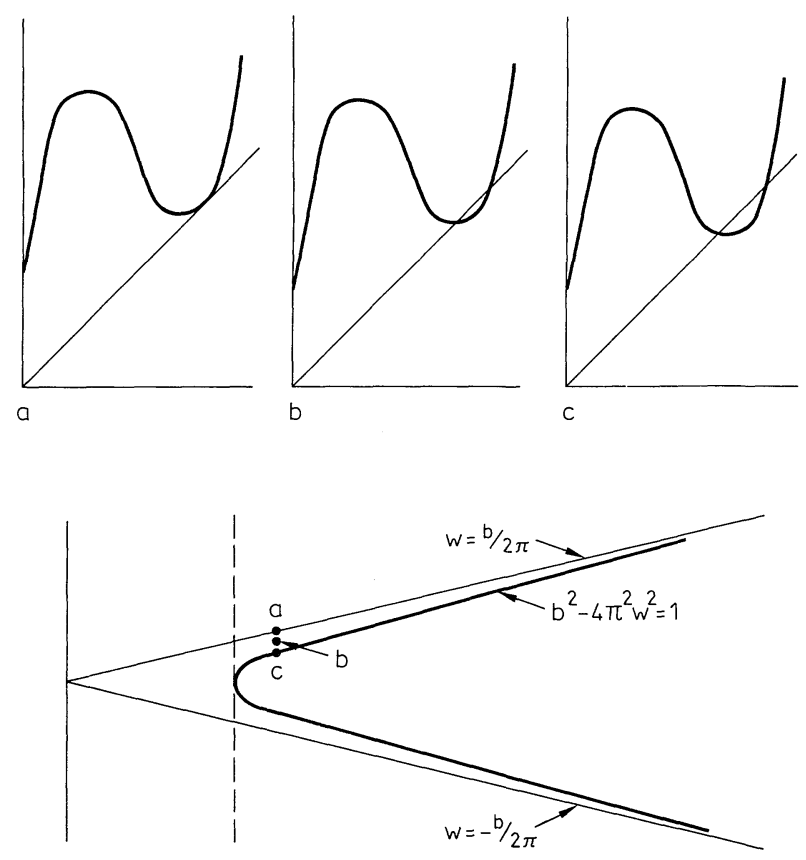

Fig. 9. Finer structure within $T_{0}$ 
$f(x)>x$ and $f(y)<y$, then $f$ has a stable fixed point in $[x, y]$. In the proof of the theorem we shall use the notation

$$
D F_{b, w}^{q}(x)=\frac{\partial F^{q}}{\partial x}(x, b, w), \quad \text { and } \quad D^{2} F_{b, w}^{q}(x)=\frac{\partial^{2} F^{q}}{\partial x^{2}}(x, b, w) .
$$

Figure 10 shows the various regions described by Theorem 4.1.

Theorem 4.1. $p$ is $C^{r}(r \geqq 1)$ and is such that Hypotheses 0 to 4 are satisfied.

(a) For each fixed rational $p / q, b$ in $[1, \infty)$ and $i=1$ or 2 , there exists a unique $w$ such that $m_{i}$ is $p / q$-periodic under $f_{b, w}$ and $o\left(m_{1}, f_{b, w}\right) \cap\left[\bar{m}_{1}, m_{1}\right)=\emptyset$ (respectively, $\left.o\left(m_{2}, f_{b, w}\right) \cap\left(m_{2}, \bar{m}_{2}\right]=\emptyset\right)$. We shall denote this $w$ by $\sigma_{i}\left(\right.$ or by $\sigma_{i, p / q}(b)$ if we need to record its dependence on $p / q$ and $b$ ).

(b) If we treat $\sigma_{i}$ as a function of $b, \sigma_{i}:[1, \infty) \rightarrow \mathbb{R}$, then $\Psi_{1}<\sigma_{1}<\Phi_{1}$ and $\Phi_{2}<\sigma_{2}<\Psi_{2}$.

(c) If $w \in\left(\sigma_{1}(b), \Phi_{1}(b)\right)$ (respectively, $w \in\left(\Phi_{2}(b), \sigma_{2}(b)\right)$ then $f_{b, w}$ has a stable p/q-periodic point $x_{w}\left(x_{w}^{\prime}\right)$ with $o\left(x_{w}, f_{b, w}\right) \cap\left(\bar{m}_{1}, m_{1}\right)=\emptyset\left(o\left(x_{w}^{\prime}, f_{b, w}\right) \cap\left(m_{2}, \bar{m}_{2}\right)=\emptyset\right)$.

(d) $\sigma_{i}:(1, \infty) \rightarrow \mathbb{R}$ is $C^{r-1}$ and further,

$$
\Gamma=\left\{\left(b, \sigma_{1}(b)\right): b \geqq 1\right\} \cup\left\{\left(b, \sigma_{2}(b): b \geqq 1\right\}\right.
$$

is $a C^{r-1}$ curve which when $r \geqq 2$ is tangent to the line $b=1$ at the point $\left(1, \sigma_{1}(1)\right)$.

Proof. Since $p / q$ is fixed throughout and $b$ is usually fixed, we shall often suppress the dependence of various quantities on them, thus $H_{1, w}=H_{1, b, w}, m_{1}=m_{1}(b)$, etc.

(a) First note that $w_{1}>w_{2}$ implies that $H_{1, w_{1}}>H_{1, w_{2}}$, and so when treated as a function of $w, H_{1, w}^{q}\left(m_{1}\right)-m_{1}$ is strictly increasing, continuous and onto. There thus exists a unique $w$ (which we denote as $\sigma_{1}$ ) with $H_{1, w}^{q}\left(m_{1}\right)=m_{1}+p .^{2}$

Next, to show that $o\left(m_{1}, h_{1}, \sigma_{1}\right) \cap\left[\bar{m}_{1}, m_{1}\right)=\emptyset$, we note that $f_{\sigma_{1}}^{q}\left(m_{1}\right)=m_{1}$, so proceeding by contradiction, assume there is an $i<q$ with $h_{1, \sigma_{1}}^{i}\left(m_{1}\right) \in\left[\bar{m}_{1}, m_{1}\right)$. But then

$$
h_{1, \sigma_{1}}^{i+1}\left(m_{1}\right)=h_{1, \sigma_{1}}\left(\left[\bar{m}_{1}, m_{1}\right)\right)=h\left(m_{1}\right),
$$

and so $h_{1, \sigma_{1}}\left(m_{1}\right)$ is $i$-periodic, a contradiction. We therefore have $o\left(m_{1}, f_{\sigma_{1}}\right)$ $=o\left(m_{1}, h_{1, \sigma_{1}}\right)$ completing part (a).

(b) Since $h_{1, \sigma_{1}}$ is nondecreasing, it has a unique rotation number which must be $p / q$ by (a). Thus $\left(b, \sigma_{1}(b)\right)$ is contained in $S_{1, p / q}$, so by the comments above Theorem 3.1, $\Psi_{1} \leqq \sigma_{1} \leqq \Phi_{1}$.

Now since $H_{1, \sigma_{1}}$ is $C^{1}$ except at $\bar{m}_{1}$ and by part (a),

$$
o\left(m_{1}, h_{1, \sigma_{1}}\right) \cap\left\{\bar{m}_{1}\right\}=\emptyset,
$$

we have that $h_{1, \sigma_{1}}^{q}$ is $C^{1}$ is a neighborhood of $m_{1}$ and by the chain rule, $D h_{1, \sigma_{1}}^{q}\left(m_{1}\right)=0$. By definition of $\sigma_{1}, H_{1, \sigma_{1}}^{q}\left(m_{1}\right)=m_{1}+p$. If we assume that $\sigma_{1}=\Phi_{1}$ then $H_{1, \sigma_{1}}^{q} \geqq R_{p}$, an impossibility. The assumption that $\sigma_{1}=\Psi_{1}$ leads to a similar contradiction.

(c) Since $\varrho\left(H_{1, \Phi_{1}}\right)=p / q$, by Lemma 2.1 we may find a point $y$ with $H_{1, \Phi_{1}}^{q}(y)$ $=y+p$ and $o(y) \cap\left(\bar{m}_{1}, m_{1}\right)=\emptyset$. Of all such $y$, let $y_{1}$ denote the $y$ which is closest to $m_{1}$ and to the right of $m_{1}$. Note that this implies that $o\left(y_{1}\right) \cap\left(\bar{m}_{1}, y_{1}\right)=\emptyset$.

\footnotetext{
${ }^{2}$ A similar continuity argument was used in [10] to show that given a $p / q$, for each $b_{0}>1$ there is at least one $w_{0}$ such that $f_{b_{0}, w_{0}}$ has a superstable $p / q$-periodic orbit
} 


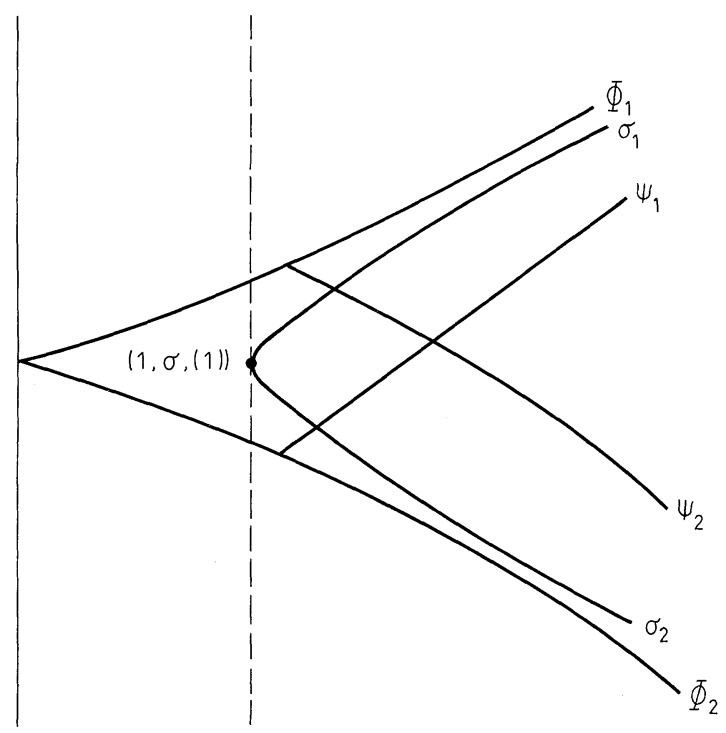

Fig. 10. Regions within $T_{p / q}$ described by Theorem 4.1

Now if $\sigma_{1}<w<\Phi_{1}$, then

$$
m_{1}+p=H_{1, \sigma_{1}}^{q}\left(m_{1}\right)<H_{1, w}^{q}\left(m_{1}\right) \leqq H_{1, w}^{q}\left(y_{1}\right)<H_{1, \Phi_{1}}^{q}\left(y_{1}\right)=y_{1}+p .
$$

Thus since $h_{1, w}$ is nondecreasing, $h_{1, w}^{q}\left[m_{1}, y_{1}\right] \subseteq\left[m_{1}, y_{1}\right]$ and by the fact above the theorem, $h_{1, w}$ has a stable $p / q$-periodic orbit $x_{w}$ in $\left[m_{1}, y_{1}\right]$.

Next, we claim that for all $x$ in $\left[m_{1}, y_{1}\right]$ and all $w$ in $\left(\sigma_{1}, \Phi_{1}\right), o\left(x, h_{1, w}\right)$ $\cap\left(\bar{m}_{1}, m_{1}\right)=\emptyset$. This will imply that $o\left(x, h_{1, w}\right)=o\left(x, f_{w}\right)$, and so $x_{w}$ is also a stable $p / q$-periodic orbit for $f_{w}$ finishing the proof.

We first prove the claim for $w=\Phi_{1}$. Since $\varrho\left(h_{1}, \Phi_{1}\right)=p / q$, one can use the same argument used in (a) to show that for $i<q, h_{1, \Phi_{1}}^{i}\left(m_{1}\right) \notin\left[\bar{m}_{1}, m_{1}\right]$. By the choice of $y_{1}$, for $i<q$, we have $h_{1, \Phi_{1}}^{i}\left(y_{1}\right) \notin\left(\bar{m}_{1}, y_{1}\right]$. Now if there was a $i<q$ with

$$
h_{1, \Phi_{1}}^{i}\left(m_{1}\right)<\bar{m}_{1}<m_{1}<y_{1}<h_{1, \Phi_{1}}^{i}\left(y_{1}\right)
$$

then $h_{1, \Phi_{1}}$ would have an $i$-periodic point in $\left[\bar{m}_{1}, y_{1}\right]$, an impossibility. We are thus left with $m_{1}<h_{1, \Phi_{1}}^{i}\left(m_{1}\right)<h_{1, \Phi_{i}}^{i}\left(y_{1}\right)<\bar{m}_{1}$ for $i<q$.

Now treated as functions of $w, h_{1, w}^{i}\left(m_{1}\right)$, and $h_{1, w}^{i}\left(y_{1}\right)$ are continuous and strictly decreasing. Since for $w$ in $\left(\sigma_{1}, \Phi_{1}\right), \varrho\left(h_{1}, w\right)=p / q$ we must have $h_{1, w}^{i}\left(m_{1}\right)$ $\neq m_{1}$ for $i<q$. We therefore have for $i<q$ and $w$ in $\left(\sigma_{1}, \Phi_{1}\right), m_{1}<h_{1, w}^{i}\left(m_{1}\right)<h_{1, w}^{i}\left(y_{1}\right)$ $<\bar{m}_{1}$. Since $h_{1, w}$ is nondecreasing and we previously showed that

$$
h_{1, w}^{q}\left(\left[m_{1}, y_{1}\right]\right) \subseteq\left[m_{1}, y_{1}\right],
$$

we have proved the claim.

(d) First, assume that $p$ is $C^{1}$. An elementary argument shows that any function $\mathbb{R} \rightarrow \mathbb{R}$ which is bounded above and below by continuous functions and has a closed graph is continuous. If $n$ is the integer with $n<p / q \leqq n+1$, then $\sigma_{1}$ is bounded above and below by the continuous functions $\Phi_{1, n+1}$ and $\Phi_{1, n}$, 
respectively. From the proof of part (a), we have that $\sigma_{1}(b)$ is the unique value of $w$ with $H_{1, b, w}^{q}\left(m_{1}\right)=m_{1}+p$. Using this, it is easy to show that $\sigma_{1}$ has a closed graph and is therefore continuous. Since $\sigma_{1}(1)=\sigma_{2}(1)$, we have that $\Gamma$ is a $C^{0}$-curve. To investigate its smoothness when $r \geqq 2$, define $L: \mathbb{R}^{3} \rightarrow \mathbb{R}^{2}$ via

$$
L_{1}(x, b, w)=F^{q}(x, b, w)-(x+p), \quad L_{2}(x, b, w)=D F(x, b, w) .
$$

Note that $L$ is $C^{r-1}$ and $L\left(m_{i}(b), b, \sigma_{i}(b)\right)=0$ for $i=1$ or 2 and $b \geqq 1$. We shall let so $L_{11}=\frac{\partial L_{1}}{\partial x}$, etc. $\quad D L=\left[\begin{array}{l}L_{11} L_{12} L_{13} \\ L_{21} L_{22} L_{23}\end{array}\right]$,

Now fix $b_{0} \geqq 1$ and $i=1$ or 2 , and let $x_{0}=m_{i}\left(b_{0}\right)$ and $w_{0}=\sigma_{i}\left(b_{0}\right)$. It is straightforward to compute the $L_{j, k}$ evaluated at $\left(x_{0}, b_{0}, w_{0}\right)$ using the fact that $o\left(x_{0}, f_{b_{0}}, w_{0}\right)$ is contained in the region where $D F_{b_{0}, w_{0}}$ is positive. Where convenient, we shall suppress the dependence of $F$ on $b_{0}$ and $w_{0}$,

$$
\begin{aligned}
& L_{11}\left(x_{0}, b_{0}, w_{0}\right)=D F^{q}\left(x_{0}, b_{0}, w_{0}\right)-1=-1, \\
& L_{12}\left(x_{0}, b_{0}, w_{0}\right)=\frac{\partial F^{q}}{\partial b}\left(x_{0}, b_{0}, w_{0}\right), \\
& L_{13}\left(x_{0}, b_{0}, w_{0}\right)=1+\sum_{j=1}^{q-1} \prod_{i=j}^{q-1} D F\left(F^{i}\left(x_{0}\right)\right)>1, \\
& L_{21}\left(x_{0}, b_{0}, w_{0}\right)=D^{2} F\left(x_{0}\right)=b_{0} p^{\prime \prime}\left(x_{0}\right) .
\end{aligned}
$$

Since $p$ satisfies Hypothesis $4, L_{21}\left(x_{0}, b_{0}, w_{0}\right)$ is zero if $b_{0}=1$ and nonzero if $b_{0}>1$.

$$
\begin{aligned}
& L_{22}\left(x_{0}, b_{0}, w_{0}\right)=\frac{\partial^{2} F}{\partial x \partial b}\left(x_{0}\right)=p^{\prime}\left(x_{0}\right) \neq 0, \\
& L_{23}\left(x_{0}, b_{0}, w_{0}\right)=\frac{\partial^{2} F}{\partial w \partial x}\left(x_{0}\right)=0 .
\end{aligned}
$$

Now if $b_{0}>1$, evaluated at $\left(x_{0}, b_{0}, w_{0}\right), L_{11} L_{23}-L_{13} L_{21} \neq 0$. Thus by the implicit function theorem, in the appropriate small neighborhoods, we have $C^{r-1}$ functions $\eta_{1}$ and $\eta_{2}$ with $(x, b, w)$ in $L^{-1}(0)$ if and only if $x=\eta_{1}(b)$ and $w=\eta_{2}(b)$. It is clear that $\eta_{2}=\sigma_{i}$.

On the other hand, evaluated at $b_{0}=1, w_{0}=\sigma_{1}(1)$ and $x_{0}=m_{1}(1), L_{11} L_{22}$ $-L_{12} L_{21} \neq 0$. We therefore have (in small neighborhoods) $C^{r-1}$ functions $\gamma_{1}$ and $\gamma_{2}$ with $(x, b, w)$ in $L^{-1}(0)$ if and only if $x=\gamma_{1}(w)$ and $b=\gamma_{2}(w)$. Evaluated at $b_{0}=1$,

$$
\frac{d \gamma_{2}}{d w}=\frac{L_{11} L_{23}-L_{13} L_{21}}{L_{11} L_{22}-L_{12} L_{21}}=0,
$$

and so $\Gamma$ is tangent to the line $b=1$.

We note that $\Gamma$ is only one branch of $L^{-1}(0)$. Other branches of $L^{-1}(0)$ which correspond to superstable $p / q$-periodic orbits with nonstandard order around the circle will be discussed elsewhere.

Because of the convergence of the edges of the $T_{r}$ given in Lemma 3.2 and the position of the splitting of $\Psi_{1, p / q}$ and $\Phi_{2, p / q}$ in the region $b>1$ given in 


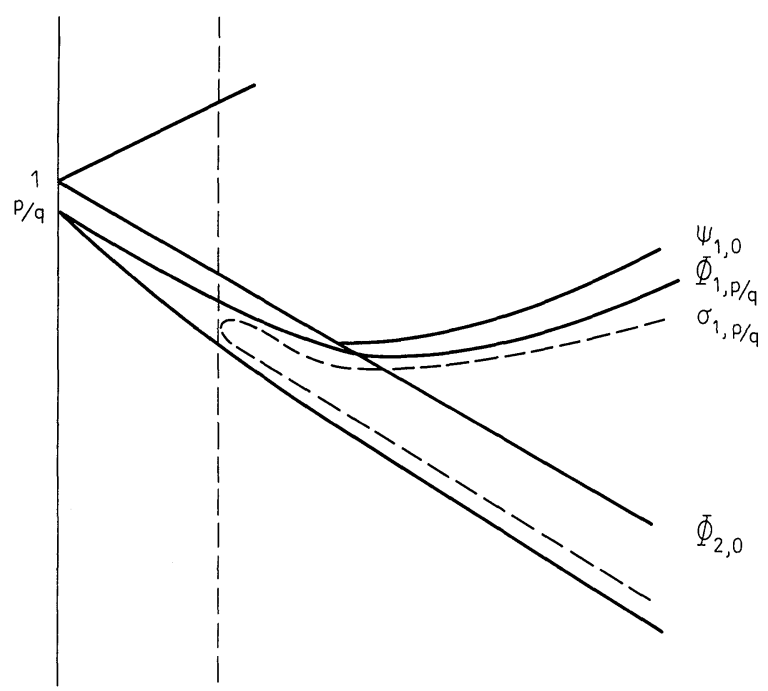

Fig. 11. An illustration of when $\sigma_{1, p / q}$ is not monotonic

Proposition 3.5, one can see that $\sigma_{i, p / q}$ is not, in general, monotonic in $x$. For example, for $p / q$ close enough to 1 , one can show that $\sigma_{1, p / q}^{\prime}$ must vanish at least twice (see Fig. 11).

Given a pair of rationals $p / q$ and $m / n$, this next corollary locates an open set which is characterized by $f_{b, w}$ having a "bistable response" of rotation number $p / q$ and $m / n$.

Corollary 4.2. $p$ satisfies Hypothesis 4. Given rationals $p / q>m / n$, there exists an open set $U$ so that $(b, w) \in U$ implies that $f_{b, w}$ has both a stable $p / q$-periodic orbit and a stable $m / n$-periodic orbit and $\varrho\left(f_{b, w}\right)=[m / n, p / q]$.

Proof. Let

$$
\begin{aligned}
U= & \left\{(b, w): \sigma_{1, m / n}(b)<w<\Phi_{1, m / n}(b)\right\} \\
& \cap\left\{(b, w): \Phi_{2, p / q}(b)<w<\sigma_{2, p / q}(b)\right\} .
\end{aligned}
$$

Using an argument as in Proposition 3.3, it is clear that $U$ is nonempty. For $(b, w) \in U, f_{b, w}$ has the appropriate properties by Theorem 4.1(b) and (c).

\section{Homoclinic Orbits and Aperiodicity}

In Sect. 3, we constructed the outline of the bifurcation diagram of our two parameter families in terms of the set $S_{i, r}$ and $T_{r}$. The boundary of $S_{i, p / q}$ consists of the graphs of the functions $\Phi_{i, p / q}$ and $\Psi_{i, p / q}$. The graphs of $\Phi_{1, p / q}$ and $\Phi_{2, p / q}$ form the boundary of $T_{p / q}$ and the graph of $\Psi_{1, p / q}\left(\Psi_{2, p / q}\right)$ is the limit of the converging upper (lower) edges of the other $T_{r}$ for $r<p / q(r>p / q)$. We showed that $f_{b, \Phi_{i, p / q}(b)}$ has a $p / q$-saddle node. In this chapter, under stronger hypotheses, we show that for $(b, w)$ on the graph of $\Psi_{1, p / q}\left(\Psi_{2, p / q}\right)$, the minimum (maximum) is a homoclinic orbit under $f_{b, w}$. 

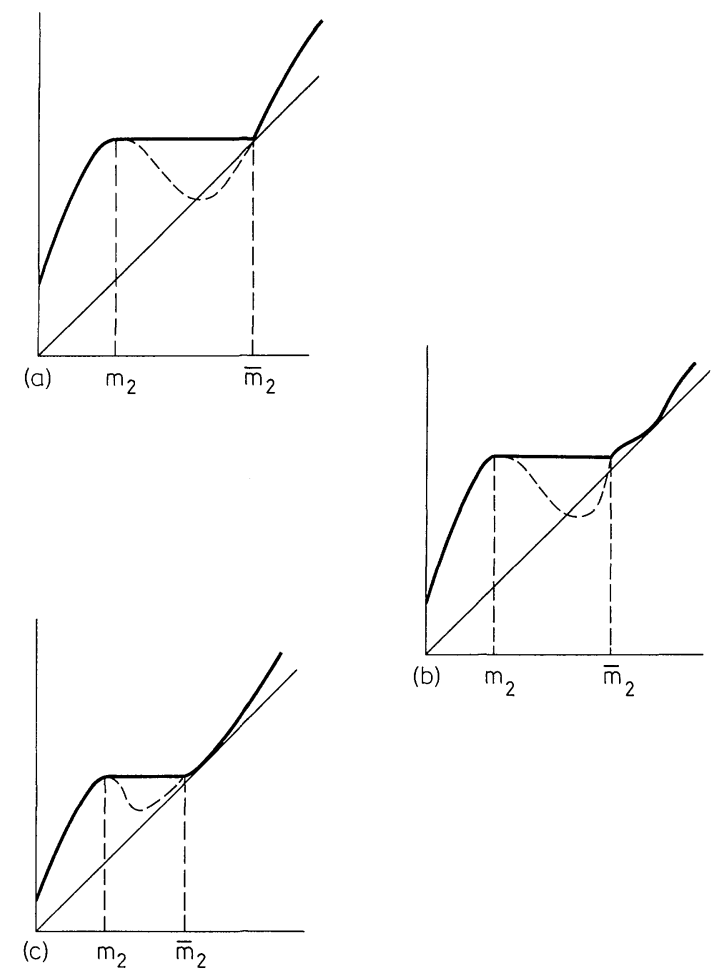

Fig. 12. Possible behavior of $f_{b, \Psi_{2,0}(b)}$

As in previous sections, we examine $T_{0}$ as the prototype for $T_{p / q}$. We recall that $\Psi_{2,0}$ is characterized by $H_{2, b, \Psi_{2,0}(b)} \geqq R_{0}$ with equality attained (see Fig. 12a). Note that $\bar{m}_{2}$ is an unstable fixed point and $F\left(m_{2}, b, \Psi_{2,0}(b)\right)=\bar{m}_{2}$. Also, $m_{2}$ has preimages arbitrarily close to $\bar{m}_{2}$. Thus $m_{2}$ 's "past" and "future" converge to $\bar{m}_{2}$. A point such as $m_{2}$ is called a homoclinic orbit (definition given below).

We wish to show that the analogous behavior happens in $T_{p / q}$. The main difficulty is illustrated in Fig. 12b. Here we still have $H_{2, b, \Psi_{2,0}(b)} \geqq R_{0}$ with equality attained but $\bar{m}_{2}$ is no longer the unstable fixed point. Such behavior is of course impossible if $f_{b, w}$ satisfies Hypothesis 4 which implies that $F_{b, w}$ has only one inflection point in $(0,1)$. Hypothesis 4 alone, however, does not seem to eliminate behavior analogous to Fig. $12 \mathrm{~b}$ happening for the $q^{\text {th }}$ iterate of $F_{b, w}$. This behavior is ruled out by requiring the function to have negative Schwarzian derivative (defined below). It is well known that if a function $G$ has negative Schwarzian, $G^{q}$ has a very nicely behaved second derivative for all integers $q$.

As has been mentioned, Fig. 12b differs from Fig. 12a in that $\bar{m}_{2}$ is not a fixed point. Using the hypothesis of negative Schwarzian, we show in Lemma 5.1 that if $\bar{m}_{2}$ is not a $p / q$-periodic point for $f_{b, \Psi_{2}(b)}$, then we are in the situation given by Proposition 3.5, i.e. $\Phi_{1}(b)=\Psi_{2}(b)$ (see Fig. 12c).

Using this, we know that when $\Phi_{1}>\Psi_{2}, \bar{m}_{2}$ is a $p / q$-periodic point under $f_{b, \Psi_{2}(b)}$. We also know that $H_{2, b, \Psi_{2}(b)}^{a} \geqq R_{p}$ with equality attained (at $\left.\bar{m}_{2}\right)$. In 
Theorem 5.2, again using the hypothesis of negative Schwarzian, we show that this implies the homoclinic behavior.

We now define the Schwarzian derivative and state some results concerning it that are contained in Singer [25]. For a $C^{3} \operatorname{map}, G: \mathbb{R} \rightarrow \mathbb{R}$, the Schwarzian derivative of $G$ at $x, S G(x)$, is defined as

$$
S G(x)=\frac{D^{3} G(x)}{D G(x)}-\frac{3}{2}\left(\frac{D^{2} G(x)}{D G(x)}\right)^{2} .
$$

(1) If $S G<0$, then $S G^{n}<0$ for all integers $n$.

(2) If $S G<0$, then $G^{\prime}$ has no negative local maximum and no positive local minimum.

(3) If $G(x) \geqq x$ in some neighborhood of $x_{0}, G\left(x_{0}\right)=x_{0}$, and $S G<0$, then $D^{2} G\left(x_{0}\right)>0$. To prove this, note that $D G\left(x_{0}\right)=1$ and if $D^{2} G\left(x_{0}\right)=0$, then since $S G<0, D^{3} G\left(x_{0}\right)<0$, which using Taylor's Theorem contradicts $G(x) \geqq x$.

(4) We will also utilize the following slight extension of Singer's theorem [25].

Theorem. $f: S^{1} \rightarrow S^{1}$ is $C^{3}$ and $F: \mathbb{R} \rightarrow \mathbb{R}$ is a lift of $f$. If $S F>0$, then for every stable $p / q$-periodic orbit $x$ of $f$, there is an $i<q$ and a critical point $c$ of $f$ so that $f^{n q}(c)$ $\rightarrow f^{i}(x)$ as $n \rightarrow \infty$.

We note as a corollary that any map $g$ is our class $A$ with $S g<0$ has at most two stable periodic orbits. One can easily compute that the canonical family $F_{b, w}$ satisfies $S F_{b, w}<0$ for all $b>1$.

The statements of Lemma 5.1 and Theorem 5.2 will only involve $\Psi_{2}$ and $\Phi_{1}$. Analogous results are of course true for $\Psi_{1}$ and $\Phi_{2}$. Also, in the statement and proof of the next lemma, we suppress dependence on $p / q$ and, in its proof, dependence on $b$. For a function $G: \mathbb{R} \rightarrow \mathbb{R}, D_{+} G(x)$ denotes the derivative of $G$ at $x$ from the right.

Lemma 5.1. If $F_{b, \Psi_{2}(b)}$ has negative Schwarzian derivative and either

(a) $H_{2, b, \Psi_{2}(b)}^{q}\left(\bar{m}_{2}\right) \neq \bar{m}_{2}+p$, or

(b) $H_{2, b, \Psi_{2}(b)}^{q}\left(\bar{m}_{2}\right)=\bar{m}_{2}+p$ and $D_{+} H_{2, b, \Psi_{2}(b)}^{q}\left(\bar{m}_{2}\right)=1$, then $\Phi_{1}(b)=\Psi_{2}(b)$.

Proof. Since $\varrho\left(H_{2, \Psi_{2}}\right)=p / q$, using Lemma 2.1(b) we may find a point $y$ with $H_{2, \Psi_{2}}^{q}(y)=y+p$ and $o\left(y, H_{2, \Psi_{2}}\right) \cap\left(m_{2}, \bar{m}_{2}\right)=\emptyset$. If we assume $(a), \bar{m}_{2} \notin o(y)$. Now since $\Psi_{2}$ is characterized by $H_{2, \Psi_{2}}^{q} \geqq R_{p}$ and $H_{2, \Psi_{2}}$ is $C^{1}$ except at $\bar{m}_{2}$, we have $D H_{2, \Psi_{2}}^{q}(y)=1$. We therefore must have $m_{2} \notin o(y)$ and so $o\left(y, H_{2, \Psi_{0}}\right) \cap\left[m_{2}, \bar{m}_{2}\right]=\emptyset$.

We may therefore find an open interval $U$ containing $y$, so $x$ in $U$ implies $F^{i}(x)$ $=H_{2}^{i}(x)$ for $i \leqq q\left(\right.$ we are omitting the subscript $\left.\Psi_{2}\right)$. Thus for $x$ in $U, F^{q}(x) \geqq x+p$ and $D F^{q}(x)=D H_{2}^{q}(x)$.

Now let $y$ denote the element of $o(y)$ which is closest to $\bar{m}_{2}$ and $y>\bar{m}_{2}$. Choose $m^{\prime}$ so that $\left(m^{\prime}, y\right]$ is the maximal interval with $\left\{x, f(x), \ldots, f^{q-1}(x)\right\} \cap\left[m_{2}, m_{1}\right]=\emptyset$ for all $x \in\left(m^{\prime}, y\right]$. Since $f$ is orientation preserving on $S^{1}-\left[m_{2}, m_{1}\right]$, it is clear that for some $i<q, f^{i}\left(m^{\prime}\right)=m_{1}$, and so $D F^{q}\left(m^{\prime}\right)=0$ (we will eventually show that $\left.m^{\prime}=m_{1}\right)$.

Next, we claim that $0<D F^{q}(x)<1$ for all $x \in\left(m^{\prime}, y\right)$. The fact that $0<D F^{q}(x)$ follows from $D F^{q}(y)=1$, continuity of $D F^{q}$ and the fact that we choose $m^{\prime}$ so that no $x \in\left(m^{\prime}, y\right]$ has $D F^{q}(x)=0$. 
To see that $D F^{q}(x)<1$, first note that we must have $D^{2} F^{q}(y)>0$ by (3) before the lemma. Now, if there was a point $x$ in $\left(m^{\prime}, y\right)$ with $D F^{q}(x) \geqq 1$, then since $D F^{q}\left(m^{\prime}\right)=0, D F^{q}$ must have a minimum at some $z \in(x, y)$. However, we have just shown that $D F^{q}(z)>0$, which contradicts the negative Schwarzian hypothesis [see (2) above the lemma].

We have proved the claim, and so for $x \in\left[m^{\prime}, y\right)$.

$$
y+p-F^{q}(x)=F^{q}(y)-F^{q}(x)=\int_{x}^{y} D F^{q}(t) d t<y-x,
$$

and so $F^{q}(x)>x+p$. Similarly, using $D F^{q}(t)>0$, we get $F^{q}(x)<y+p$. Thus

$$
f^{a}\left(\left[m^{\prime}, y\right]\right) \cong\left[m^{\prime}, y\right] .
$$

But since $\left\{x, f(x), \ldots, f^{q-1}(x)\right\} \cap\left[m_{2}, m_{1}\right]=\emptyset$ for all $x$ in $\left(m^{\prime}, y\right]$, we have $o(x)$ $\cap\left[m_{2}, m_{1}\right]=\emptyset$, and so $f^{i}$ restricted to $\left[m^{\prime}, y\right]$ is a homeomorphism for all $i$.

Next, we show that in fact, $m^{\prime}=m_{1}$. If $m^{\prime} \neq m_{1}$, then since $y$ is the minimum on its orbit and $f^{i}\left(m^{\prime}\right)=m_{1}$, we have $m^{\prime}$ interior to $f^{i}\left(\left(m^{\prime}, y\right)\right)$, and so $m^{\prime \prime}=f^{-i}\left(m^{\prime}\right)$ is interior to $\left(m^{\prime}, y\right)$ and $f^{2 i}\left(m^{\prime \prime}\right)=m_{1}$, a contradiction to the choice of $m^{\prime}$.

Summarizing what we have so far, $x \in\left[m_{1}, y\right)$ implies that $F^{q}(x)>x+p$. Now for $x^{\prime} \in\left[\bar{m}_{1}, m_{1}\right]$, let $x$ be the point in $\left[m_{1}, \bar{m}_{2}\right] \subseteq\left[m_{1}, y\right]$ with $F(x)=F\left(x^{\prime}\right)$. Then since $x>x^{\prime}, F^{q}\left(x^{\prime}\right)=F^{q}(x)>x+p>x^{\prime}+p$. Thus $x \in\left[\bar{m}_{1}, y\right)$ implies that $F^{q}(x)$ $>x+p$.

Finally, say there is a $z$ in $\mathbb{R}$ with $F^{q}(z)<z+p$. Then, since $H_{2}^{q} \geqq R_{p}$, there must be some $i \leqq q$ with $F^{i}(z) \neq H_{2}^{i}(z)$. If we choose the least such $i$, then $f^{i}(z) \in\left(m_{2}, \bar{m}_{2}\right)$. Now since $\mathrm{H}_{2}$ is order preserving and $F \leqq H_{2}, F^{q}(z)<z+p$ implies that

$$
F^{q}\left(F^{i}(z)\right)=F^{i}\left(F^{q}(z)\right) \leqq H_{2}^{i}\left(F^{q}(z)\right) \leqq H_{2}^{i}(z+p)=H^{i}(z)+p=F^{i}(z)+p .
$$

This contradicts the result we summarized above. Thus $F_{\Psi_{2}}^{q} \geqq R_{p}$ with equality attained so by the comment after Lemma 2.5, $\Psi_{2}=\Phi_{1}$. If one assumes $(b)$, the proof is similar.

We shall again suppress the dependence on $p / q$.

Theorem 5.2. Fix $b>1$. If $\Phi_{1}(b)>\Psi_{2}(b)$ and $F_{b, \Psi_{2}(b)}$ has negative Schwarzian derivative, then $\bar{m}_{2}$ is an unstable p/q-periodic point under $f_{b, \Psi_{2}(b)}, f_{b, \Psi_{2}(b)}^{q}\left(m_{2}\right)=\bar{m}_{2}$ and for each open interval $U$ containing $\bar{m}_{2}$, there is an $i$ with $m_{2} \in f^{i}(U)$.

Proof. Theorem 3.1(b) says that $\Phi_{1}(b) \geqq \Psi_{2}(b)$. Using Lemma 5.1, $\Phi_{1}(b)>\Psi_{2}(b)$ implies that $H_{2}^{q}, \Psi_{2}\left(\bar{m}_{2}\right)=\bar{m}_{2}+p$ and $D_{+} H_{2}^{q}, \Psi_{2}\left(\bar{m}_{2}\right) \neq 1$ (we are omitting the dependence on $b$ ). Now since $H_{2, \Psi_{2}}^{q} \geqq R_{p}$, it is clear that $D_{+} H_{2, \Psi_{2}}^{q}\left(\bar{m}_{2}\right)>1$ and $o\left(\bar{m}_{2}, H_{2, \Psi_{2}}\right)=o\left(\bar{m}_{2}, F_{\Psi_{2}}\right)$, and so $D F_{\Psi_{2}}^{q}\left(\bar{m}_{2}\right)>1$ and $\bar{m}_{2}$ is an unstable periodic orbit under $f_{\Psi_{2}}$. Since $f_{\Psi_{2}}\left(m_{2}\right)=f_{\Psi_{2}}\left(\bar{m}_{2}\right)$, we have $\bar{m}_{2}=f_{\Psi_{2}}^{q}\left(\bar{m}_{2}\right)=f_{\Psi_{2}}^{q}\left(m_{2}\right)$.

To prove the rest of the theorem we examine $H_{2, \Psi_{2}}$ more closely (we omit the subscript $\Psi_{2}$ ). Since $\varrho\left(H_{2}\right)=p / q$, if we let $I=\left[m_{2}, \bar{m}_{2}\right]$ and $I_{-i}=H_{2}^{-i}(I)$, then using an argument as in Lemma 2.1(b) we have that the sets $I, I_{-1}, \ldots, I_{-(q-1)}$ are pairwise disjoint closed intervals and $f^{i}$ restricted to $I_{-i}$ is a homeomorphism onto $I$ for $0 \leqq i \leqq 1$. Moreover, if

then $H_{2}^{i}(x)=F^{i}(x)$ for $i \leqq q$.

$$
x \notin \bigcup_{i=0}^{q-1} I_{-i}
$$


Now choose $j$ so that $I_{-j}$ is the next $I_{-i}$ to the right of $I$. Let $I_{-j}=\left[m_{2}^{\prime}, \bar{m}_{2}^{\prime}\right]$, and so $f^{j}\left(m_{2}^{\prime}\right)=m_{2}$. We now show that for $x$ in $\left(\bar{m}_{2}, m_{2}^{\prime}\right), F^{q}(x)>x+p$. Since $\left(\bar{m}_{2}, m_{2}^{\prime}\right)$ is in the complement of the $I_{-i}$ 's, we know that $x \in\left(\bar{m}_{2}, m_{2}^{\prime}\right)$ implies that $H_{2}^{q}(x)$ $=F^{q}(x)$. Since by definition, $H_{2}^{q} \geqq R_{p}$, we have $F^{q}(x) \geqq x+p$ for all $x$ in $\left(\bar{m}_{2}, m_{2}^{\prime}\right)$.

Now, if there was a point $z$ in $\left(\bar{m}_{2}, m^{\prime}\right)$ with $F^{q}(z)=z+p$, then $D F^{q}(z)=1$, and so by (3) above Lemma 5.1, we have $D^{2} F^{q}(z)>0$. Since $D F^{q}\left(\bar{m}_{2}\right)>1, D F^{q}$ must have a local minimum at some point $x$ in $\left(\bar{m}_{2}, z\right)$. But every point $x$ in $\left(\bar{m}_{2}, m_{2}^{\prime}\right)$ satisfies $\left\{x, f(x), \ldots, f^{q-1}(x)\right\} \cap\left[m_{2}, \bar{m}_{2}\right]=\emptyset$, and so $D F^{q}(x)>0$. This contradicts the negative Schwarzian hypothesis. We therefore have $F^{q}(x)>x+p$ for all $x$ in $\left(\bar{m}_{2}, m_{2}^{\prime}\right)$.

Now let $U$ be an open interval containing $\bar{m}_{2}$. Since $F^{q}\left(\bar{m}_{2}\right)=\bar{m}_{2}+p$ and we have just shown that $F^{q}(x)>x+p$ for all $x$ in $\left(\bar{m}_{2}, m_{2}^{\prime}\right)$, it is clear that we can find a $k$ with $m_{2}^{\prime} \in f^{k q}(U)$. But $f^{j}\left(m_{2}^{\prime}\right)=m_{2}$ and so $m_{2} \in f^{k q+j}(U)$, completing the proof of the theorem.

Loosely speaking, a homoclinic orbit is one whose past and future converge to the same point or periodic orbit. They result in rich dynamical behavior and have been a central object of study in dynamical systems since they were first introduced by Poincaré. Homoclinic points for maps of the interval $f:[0,1] \rightarrow[0,1]$, were first defined by $L$. Block [4]. A point $x$ is called homoclinic to the $q$-periodic point $z$ if $x \neq z, f^{m q}(x)=z$ for some $m$, and given an open interval $U$ containing $z$, there must exist an $n$ with $x \in f^{n q}(U)$. In other words, one requires that preimages and iterates of $x$ under $f^{q}$ come arbitrarily close to $z$.

In Theorem 5.2 we proved that preimages of $m_{2}^{\prime}$ under $f^{q}$ come arbitrarily close to $\bar{m}_{2}$. This means that preimages of $m_{2}$ under $f^{q}$ come arbitrarily close to $f^{j}\left(\bar{m}_{2}\right)$ and of course, $f^{q}\left(m_{2}\right)=\bar{m}_{2}$. Thus under $f^{q}$, the past and future of $m_{2}$ go to different points on the orbit of $\bar{m}_{2}$, and so $m_{2}$ is not homoclinic to $\bar{m}_{2}$ in Block's sense. One could interpret Theorem 5.2 as saying that $m_{2}$ is a homoclinic point for the periodic orbit that contains $\bar{m}_{2}$. One could also borrow terminology from the theory of invertible dynamical systems and say that under $f^{q}, m_{2}$ is heteroclinic between two different iterates of $\bar{m}_{2}$.

It is interesting to note that similar considerations occur in [2] in the discussion of the curve in the resonance horn that is analogous to the graph of $\Psi_{2, p / q}$. The fact that the maximum $m_{2}$ is the homoclinic point takes the analogy a step further as it seems to correspond to a homoclinic tangency.

The results of Sect. 3 and Theorem 5.2 allow us to give a nice characterization of the dynamics of $f_{b, w}$ as it makes the transition from having a single rotation number to having an interval of rotation numbers. The various cases are illustrated in Fig. 13. I suspect that the small island that occurs in $T_{p / q}$ cannot actually occur, but none of my results prove this.

Corollary 5.3. $f_{b, w}$ has negative Schwarzian derivative for all $b>1$ and $(b, w) \in B d\left(\left\{(b, w): \varrho\left(f_{b, w}\right)\right.\right.$ is a single number $\left.\}\right)$.

(a) If $\varrho\left(f_{b, w}\right)$ is an irrational $\alpha$, then $b=1$ and $w=\Phi_{1, \alpha}(1)=\Phi_{2, \alpha}(1)$.

(b) If $\varrho\left(f_{b, w}\right)=p / q$ and

(i) $(b, w) \in B d\left(T_{p / q}\right)$, then $f_{b, w}$ has a $p / q$-saddle node, or

(ii) if $(b, w) \notin B d\left(T_{p / q}\right)$, then either $m_{1}$ or $m_{2}$ is homoclinic to an unstable $p / q$ periodic orbit. 


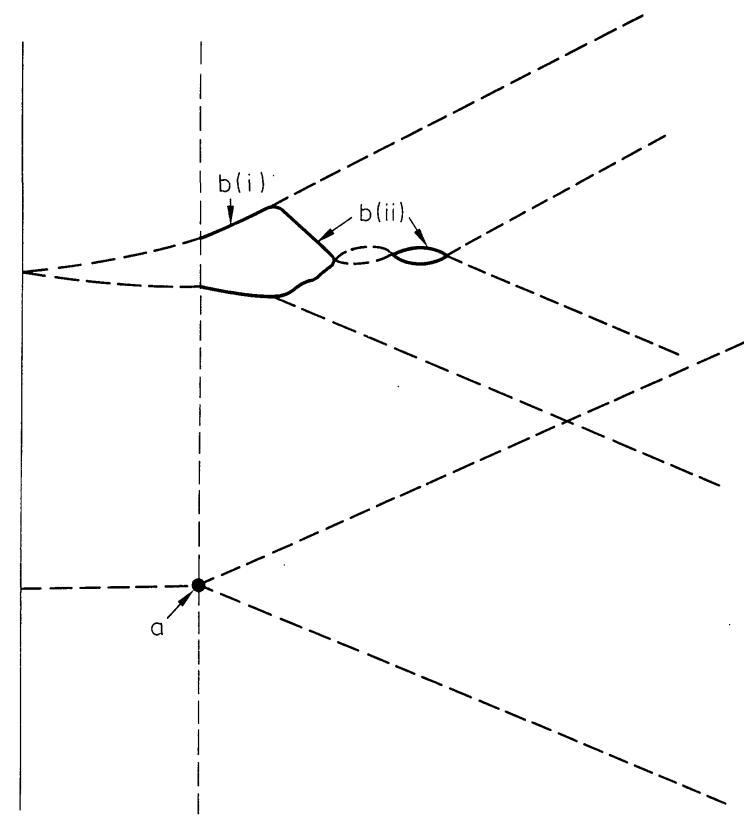

Fig. 13. The boundary of the region where $f_{b, w}$ has a unique rotation number

The next corollary locates an uncountable set of parameter values $(b, w)$ for which $f_{b, w}$ is "aperiodic."

Corollary 5.4. $\alpha$ and $\beta$ are irrational numbers with $\alpha>p / q>\beta>m / n$ and $F_{b, w}$ has negative Schwarzian derivative for $b>1$. If $b$ and $w$ are such that they satisfy any of the following equalities, then $f_{b, w}$ has no stable periodic orbits.
(a) $w=\Phi_{2, \alpha}(b)=\Phi_{1, \beta}(b)$,
(b) $w=\Psi_{2, p / q}(b)=\Phi_{1, \beta}(b)$,
(c) $w=\Psi_{1, p / q}(b)=\Phi_{2, \alpha}(b)$,
(d) $w=\Psi_{1, m / n}(b)=\Psi_{2, p / q}(b)$,
(e) $w=\Psi_{1, p / q}(b)=\Psi_{2, p / q}(b)$.

Proof. Note that all these cases do occur by Proposition 3.3. We prove the conclusion for case (b), the others are similar. By Theorem 5.2, $f_{b, w}^{q}\left(m_{2}\right)$ is an unstable periodic orbit and by Proposition 2.3, $\varrho\left(m_{1}\right)=\alpha$, an irrational number. Thus neither of the critical points is attracted to a stable periodic orbit, and so by Singer's Theorem, $f_{b, w}$ has none.

We note that if $g$ is a map in the class $A$ discussed in Sect. 2 and $G$ has negative Schwarzian derivative, then $\varrho(g)=[\alpha, \beta]$ implies by Proposition 2.3 that $\varrho\left(m_{1}\right)=\alpha$ and $\varrho\left(m_{2}\right)=\beta$, and so $g$ has no stable periodic orbits.

In the last proposition we examine the smoothness of the $\Phi_{i}$ when $f_{b, w}$ has negative Schwarzian.

Proposition 5.5. If $f_{b, w}$ is $C^{r}$ with $r \geqq 3$ and $F_{b, w}$ has negative Schwarzian for all $b>1$, then $\Phi_{i}:(1, \infty) \rightarrow \mathbb{R}$ is $C^{r-1}$ for $i=1,2$. 
Proof. The proof is quite similar to that of Theorem 4.1(d), so we just give a sketch. Fix $p / q$ and $b_{0}>1$. Recall that $\Phi_{1}\left(b_{0}\right)$ is characterized by $F_{\Phi_{1}}^{q} \geqq R_{p}$ with equality attained at some $y_{0}$ with $D F^{k}\left(y_{0}\right)>0$ for all $k$ [see the proof of Theorem 4.1(c) $]$. We also have that $D F^{q}\left(y_{0}\right)=1$, and so by (3) above Lemma $5.1, D^{2} F^{q}\left(y_{0}\right)>0$.

Now define $M: R^{3} \rightarrow R^{2}$ via

$$
M_{1}(x, b, w)=F^{q}(x, b, w)-(x+p), \quad M_{2}(x, b, w)=D F^{q}(x, b, w)-1 .
$$

Note that $M$ is $C^{r-1}$, and if we let $w_{0}=\Phi_{1}\left(b_{0}\right)$, then $M\left(y_{0}, b_{0}, w_{0}\right)=0$.

One computes that, evaluated at $\left(y_{0}, b_{0}, w_{0}\right), M_{11}=0, M_{13}>1$, and $M_{21}=D^{2} F^{q}\left(y_{0}\right) \neq 0$. Thus $M_{11} M_{23}-M_{21} M_{13} \neq 0$, and we are done by the Implicit Function Theorem.

Acknowledgements. This paper is essentially the author's thesis and he would like to thank his advisor, Alan Dankner, for his support and encouragement. Thanks also to Dick Hall, for several very useful conversations and, in particular, for pointing out the construction in Sect. 2 which considerably simplified the proofs of many of these results.

\section{References}

1. Arnol'd, V.I.: Small denominators. I. On the mappings of the circumference onto itself. Trans. Am. Math. Soc. $2^{\text {nd }}$ Ser. 46, 213 (1965)

2. Aronson, D., Chory, M., Hall, G., McGehee, R.: Bifurcation from an invariant circle for two parameter families of maps of the plane: a computer assisted study. Commun. Math. Phys. 83, 303 (1982)

3. Bernhardt, C.: Rotation intervals of a class of endomorphisms of the circle. Proc. Lond. Math. Soc., III Ser. 45 part 2 (1982)

4. Block, L.: Homoclinic points of mappings of the interval. Proc. Am. Math. Soc. 72, 3, 576-580 (1978)

5. Block, L., Franke, J.: Existence of periodic points for maps of $S^{1}$. Invent. Math. 22, 69-73 (1973)

6. Brunovsky, P.: Generic properties of the rotation number of one parameter diffeomorphisms of the circle. Czech. Math. J. 24 (99),74-90 (1974)

7. Collet, P., Eckmann, J.-P.: Iterated maps on the interval as dynamical systems. Boston: Birkhäuser 1980

8. Coullet, P., Tresser, C., Arneodo, A.: Transition to turbulence for doubly periodic flows. Phys. Lett. 77A, (5), 327-331 (1980)

9. Feigenbaum, M., Kadanoff, L., Shenker, S.: Quasiperiodicity in dissipative systems: a renormalization group analysis. Physica 5D, 370-382 (1982)

10. Glass, L., Guevara, M., Shrier, A., Perez, R.: Bifurcation and chaos in a periodically stimulated cardiac oscillator. Proc. of the Los Alamos Conf. on "Order in Chaos," Physica 7D, 89-103 (1983)

11. Greenspan, B., Holmes, P.: Repeated resonance and homoclinic bifurcation in a periodically forced family of oscillators. SIAM J. Math. Anal. (to appear)

12. Hall, G.: A $C^{\infty}$ Denjoy counterexample. Ergodic Theory Dyn. Syst. 1, 261-272 (1981)

13. Hall, G.: Personal communication, 1983

14. Hall, G.: Resonance zones in two-parameter families of circle homeomorphism. MRC Technical Summary Report, May, 1983

15. Herman, M.: Measure de Lebesque et nombre de rotation. Lecture Notes in Math. Vol. 597. Berlin, Heidelberg, New York: Springer 1977

16. Herman, M.: Sur la conjugaison differentiable des diffeomorphismes du cercle des rotations. Publ. Math., Inst. Hautes Etud. Sci. 49, 5-234 (1979) 
17. Hoppensteadt, F., Keener, J.: Phase locking of biological clock. J. Math. Biol. 15, 339-349 (1982)

18. Ito, R.: Rotation sets are closed. Math. Proc. Camb. Philos. Soc. 89, 107-111 (1981)

19. Jensen, M., Bak, P., Bohr, T.: Complete devils staircase, fractal dimension, and universality of mode-locking structure in the circle map. Phys. Rev. Lett. 50, 1637-1639 (1983)

20. Kadanoff, L.: Supercritical behavior of an ordered trajectory. J. Stat. Phys. 31, 1-27 (1983)

21. Newhouse, S., Palis, J., Takens, F.: Bifurcations and stability of families of diffeomorphisms. Publ. Math., Inst. Hautes Etud. Sci. 57, 5-72 (1983)

22. Ostlund, S., Rand, D., Sethna, J., Siggia, E.: Universal properties of the transition from quasiperiodicity to chaos in dissipative systems. Physica 8D, 303-342 (1983)

23. Perez, R., Glass, L.: Bistability period doubling bifurcations and chaos in a periodically forced oscillator. Phys. Lett. 90A, 441-443 (1982)

24. Shenker, S.: Scaling behavior in a map of a circle onto itself: empirical results. Physica 5D, 405-408 (1982)

25. Singer, D.: Stable orbits and bifurcations of maps of the interval. SIAM J. Appl. Math. 35, 260-267 (1978)

Communicated by O. E. Lanford

Received March 5, 1984 
